QUESTION i. Does a Hausdorff linear-compact relatively complemented lattice with zero have a unit 1 ?

QUESTION ii. Is the lattice of congruences of a discrete linear-compact C*-lattice finite?

In this connection I wish to express my gratitude to Doctor V. S. Krishnan and Doctor V. K. Balachandran for their valuable guidance in the preparation of this paper. My thanks are also due to the referee for suggesting various improvements (particularly (3.14)).

\title{
References
}

[1] V. K. Balachandran, Stone's topologization of the distributive lattice, Doctorate dissertation, University of Madras, 1950.

[2] G. Birkhoff, Lattice theory, New York 1948.

[3] Dilworth, The structure of relatively complemented lattices, Ann. of Math. 51 (1950), pp. 348-359.

[4] Ha shimoto, Ideal theory for lattices, Math. Japonicae 2 (1952), pp. 149-186.

[5] J. L. Kelley, General topology, 1957.

[6] S. Lefschetz, Algebraic topology, Amer. Math. Soc. Coll. Publ. 1942.

[7] H. Leptin, Über eine Klasse linear-kompakten abelschen Gruppen. 1, Abhand. Yrath. Sem. Unir. Hamburg B-19 (1954-55), pp. 23-40.

[8] F. Maeda, Kontinuierliche Geometrien, Springer, Berlin 1958.

[9] P. S. Rema, On sets, lattices and groups with Boolean metrization, Doctorate dissertation, University of Madras, 1963.

DEPARTMENT OF MATHEMATICS AND RAMANUJAN INSTITUTE UNIVERSITY OF MADRAS

Reçu par la Rédaction le 11. 4. $190 t$

\section{On a singular plane continuum *}

by

\section{R. Dud a (Wrocław)}

§1. Introduction. Using slightly extended Bernstein's argument on the decomposition of a plane into two disjoint and totally imperfect ${ }^{(1)}$ subsets (cf. [3], p. 422), it is easy to decompose each complete separable space $I$ having the property:

(1) if a set $A \subset Y$ separates $I$, then $A$ contains a perfect subset,

into a countable sequence of disjoint, connected, ponctiform $\left(^{2}\right)$ and dense subsets. Such are, for instance, all manifolds (in particular, euclidean spaces) of dimension $n \geqslant 2$, the universal curve of Sierpiński ("a carpet"; see [4], p. 202) and many others. The points of these spaces are of continuumrange.

On the other hand, however, such a decomposition is impossible for a regular curve $\left(^{3}\right)$. Moreover, a regular curve even does not contain a countable sequence of disjoint and connected sets $\left\{S_{k}\right\}_{k=1,2, \ldots}$ of diameter $\left.\delta\left(S_{k}\right)>\varepsilon>0{ }^{4}\right)$. Thus a natural question arises whether decomposition:

(2) $X=\bigcup^{\infty} S_{k}$, where $S_{k}$ are mutually disjoint, connected, ponctiform and dense subsets of $X$

(hence of diameter $\delta\left(S_{k}\right)=\delta(X)$ ), is possible for a continuum $X$ not possessing property (1)? Is it possible for a rational curve ${ }^{(5)}$, which,

* This research was partially supported by the D. S.I.R.

(1) A subset $A$ of a space $I$ is said to be tocally imperfect provided that it does not contain any perfect subset of $Y$ (cf. [3], p. 421).

(2) A set $A$ is said to be ponctiform provided tnat each of its subcontinua consists of one point only (cf. [4], p. 130).

(3) A continuum $Y$ is said to be regular curve provided that each its point is of finite or $\omega$ range or, in ouher words, that each its point has arbitrarily small neighbourhoods, the boundaries of which are finite ([4], p. 201). In particular, $\operatorname{dim} Y \leqslant 1$.

(4) For suppose that a regular curve $Y$ does. As a compact, it contains then a point $p \in Y$ such that each neighbourhood $G$ of $p$ meets infinitely many $S_{k}$. Taking $G$ of diameter $\delta(G)<\varepsilon$, we have, by our assumption and connectedness of $S_{k}$, $\operatorname{Fr}(G) \cap S_{k} \neq 0$ for infinitely many $S_{k}$, and therefore $\operatorname{Fr}\left(S_{k}\right)$ must be infinite (sets $S_{k}$ are disjoint). A contradiction.

(5) A continuum $Y$ is said to be a rational ourve provided that each its point is of at most countable range or, in other words, that each point has arbitrarily small neighbourhoods, the boundaries of which are finite or countable ([4], p. 201). 
by its definition, can be separated between each two points by a finite or countable subset?

The aim of this paper is to prove that there exists $\left(^{6}\right)$ a continuum $X$ which has the property (1) and is not only rational but even hereditarily locally connected $\left(^{7}\right.$ ) and plane (see Theorems 1 and 2 ), that is, from topological point of view, the simplest one among rational but not regular curves.

This continuum $X$ may be also interesting with a respect to a wellknown theorem of Gehman, who has proved (see [4], p. 366) that hereditarily locally connected and plane continua are characterized by not containing any sequence of disjoint subcontinua of diarpeter surpassing any positive value given in advance. This result was completed by Urysohn and Whyburn (cf. [4], p. 196), who constructed a spatial but hereditarily locally connected continuum containing such a sequence. The result of Gehman was later improved by Lelek, who has shown [5] that hereditarily locally connected and plane continuum does not contain any sequence of connected subsets, any two of which are separated and all of which have diameters surpassing any positive value given in advance. This raises a natural question whether hypothesis of separatedness in Lelek's theorem is essential or can it be replaced by a weaker one that sets in question are mutually disjoint only? The answer is given by the continuum $X$ : there exists hereditarily locally connected and plane continuum which not only contains but simply is composed of a countably many connected, mutually disjoint and of diameter $\delta(X)$ subsets. (They are, moreover, dense and ponctiform.)

The continuum $X$ may be also regarded as a some kind of generalization of a construction given by Knaster and Kuratowski, who have established [2] in a Triangle Curve of Sierpiński a connected, locally connected and ponctiform subset. Namely, each set $S_{k}$ is, as a subset of hereditarily locally connected continuum $X$, locally connected itself (cf. [4], p. 199) and therefore the main result of the paper (see Theorems 1 and 2 ) can be restated as follows:

THEOREM 0. There exists a sequence $\left\{S_{k}\right\}_{k=1,2, \ldots}$ of connected, locally connected, disjoint and ponctiform subsets of plane, the closure each of which is the same hereditarily locally connected continuum $X$.

The definition of $X$ is unfortunately rather complicated. It is, in some sense, similar to the mentioned above Triangle Curve and will be

(6) Strictly speaking, the paper describes a method of construction of such a continuum. Since construction is not univocal (see reference on p. 32), then there is many (probably $2^{k_{0}}$ ) hereditarily locally connected and plane continua satisfying (2).

( ${ }^{7}$ A continum $X$ is said to be hereditarily locally connected provided that each subcontinuum of $X$ is locally connected ([4], p. 195). In particular, if $X$ is hereditarily locally connected, then $X$ is rational ([6], p. 94) and therefore I-dimensional. Full account of ths property can be found in [4] and [6]. obtained in a similar procedure by removing open dises from a triangle (roughly speaking, more but smaller ones). In view of technical difficulties we must, however, use the machinery of upper semicontinuous decompositions. Nevertheless, the very idea of the construction is simple and will be fully explained in $\S 3$. The proof of hereditarily locally connectedness is intuitively obvious and short. It is given in $\S 4$.

And as to the proof of property (2), let us mention its main features. We shall distinguish in $X$ a countable set $V$, which we shall divide next into a countable sequence of mutually disjoint subsets $V_{k}$ each of which has the property

(3) if set $A \subset X$ separates $X$ and $X \cap V_{k}=0$ for some $k$, then $A$ contains a perfect subset.

(compare in with (1)). The proof of this property takes the most part of the paper and is rather difficult. But when it is done, the rest is simple. Following the mentioned Bernstein's argument we divide $X-V$ into a countable sequence $\left\{\boldsymbol{H}_{n}\right\}_{n=1,2, \ldots}$ of mutually disjoint and totally imperfect subsets. Since each separator of $X$ missing some $V_{k}$ must meet by (2) the set $H_{k}$, then $H_{k} \cup V_{k}$ is connected for each $k$ and it suffices to put $S_{k}=H_{k} \cup V_{k}$.

I am obliged to Professor B. Knaster for the problem and a good deal of helpful suggestions and criticism, to which I owe so much.

\$2. Construction. Let $N_{k}$, where $k$ is natural, denote all sequences consisting of $k$ members any one of which is equal to one of the numbers $0,1,2$ or 3 . The elements of $N_{k}$ will be used in sequel as indices of the sets and will be denoted by $v_{k}, \mu_{k}, \pi_{k}$, or $\tau_{k}$. The number $k$ will be called an order of the set provided with such an index. In the case of order 0 which also will be used in this paper, i.e. for $k=0$, we simply omit the index.

The continuum $X$ will be defined as the hyperspace of some upper semicontinuous decomposition of other continuum $\widetilde{X}$.

We shall proceed gradually starting with the operation on $\widetilde{T}_{v_{k}}$, where $\widetilde{T}_{v_{k}}$ is an arbitrary at the moment but equilateral triangle. Let $a$ be the length of the side of $\widetilde{T}_{y_{k}}$. At a distance $a / 3$ from each vertex take a point on any of two sides ending at that vertex and join them by a segment.

Similarly, join by segments also the middle points of the sides of $\widetilde{T}_{v_{k}}$. In this way the triangle $\widetilde{T}_{v_{k}}$ is now divided into 4 equilateral triangles (on which the operation $\gamma$ will be iterated) and 3 trapesoids (see Fig. 1). Denote the central triangle by $\widetilde{T}_{v_{k} 0}$ and the remaining triangles by $\widetilde{T}_{v_{k 1} 1}, \widetilde{T}_{v_{k} 2}$ and $\widetilde{T}_{v_{k}}$; everyone of these 4 triangles is now of order $k+1$. By $\widetilde{P}_{v_{k}}^{i}$ denote trapesoid having a common side with a triangle $\widetilde{T}_{v_{k} i}$; this side of $\widetilde{P}_{v_{k}}^{i}$ and the opposite one will be called conjugate. They play 
an important role in the sequel. The remaining sides of the trapesoids $\widetilde{P}_{v_{k}}^{i}$ form 3 middle segments (in partition for 3 equal parts according to our definition of the operation $\gamma$ ) of the sides of the triangle $\widetilde{T}_{\nu_{v}}$. These segments will be called completive and their union will be denoted by $\widetilde{L}_{y}$.

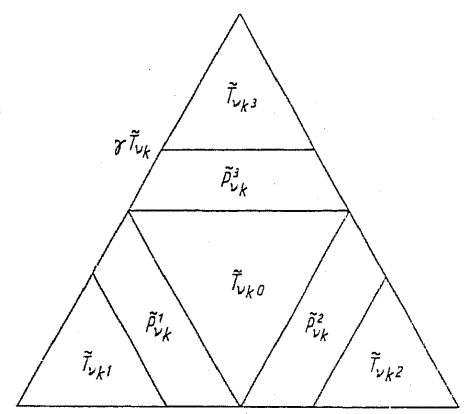

Fig. 1

By $\gamma \widetilde{T}_{v_{k}}$ we shall mean the continuum $\widetilde{L}_{\nu_{k}} \cup \bigcup_{i=0}^{3} \widetilde{T}_{\nu_{k} i}$, i.e. the continuum made of the triangle $\widetilde{T_{v}}$ by removing from it the interiors of 3 trapesoids $\widetilde{P}_{\boldsymbol{v}_{k}}^{1}, \widetilde{P}_{\boldsymbol{v}_{k}}^{2}$ and $\widetilde{P}_{\boldsymbol{v}_{k}}^{3}$.

Using now just circumscribed operation $\gamma$ we can define a decreasing sequence of continua $\left\{\widetilde{Y}_{k}\right\}_{k=1,2, \ldots}$.

Let $\widetilde{T}$ be the equilateral triangle of vertices $(0,0),(1,0)$ and $\left(\frac{1}{2}, \sqrt{3} / 2\right)$. Put $\widetilde{Y}_{1}=\gamma \widetilde{T}$ (see Fig. 2, but at the moment do not pay attention to arrows). Hence $\widetilde{Y}_{1}$ is a continuum consisting of 4 triangles $\widetilde{T}_{0}, \widetilde{T}_{1}, \widetilde{T}_{2}, \widetilde{T}_{3}$ and joining them 3 completive segments of order 0 (the union of which, according to our convention of omitting an index $v_{0}$, is denoted by $\widetilde{L}$ ). Defining now $\widetilde{Y}_{2}$ by the application of the operation $\gamma$ to any of the 4 triangles of $\widetilde{Y}_{1}$ we receive the continuum consisting of $4^{2}$ triangles $\widetilde{T}_{00}, \widetilde{T}_{01}, \ldots, \widetilde{T}_{33}$ and joining them completive segments of orders 0 and 1 , belonging to the sets $\widetilde{L}, \widetilde{L}_{0}, \widetilde{L}_{1}, \widetilde{L}_{2}, L_{3}$ (see Fig. 3 , but at the moment do not pay attention to the arrows). We proceed by induction putting for $k=1,2, \ldots$

$$
\widetilde{Y}_{k+1}=\bigcup_{v_{k} \in N_{k}} \gamma \widetilde{T_{v_{k}}} \cup \bigcup_{l=0}^{k-1} \bigcup_{\nu_{l} \in N_{l}} \tilde{L_{\nu_{l}}}
$$

Hence, by the definition of $\gamma \widetilde{T_{\boldsymbol{p}_{k}}}$, we have

$$
\widetilde{\Upsilon}_{k}=\bigcup_{\boldsymbol{p}_{k} \in N_{k}} \widetilde{T}_{\boldsymbol{y}_{k}} \cup \bigcup_{l=0}^{k=1} \bigcup_{\eta_{l} \in N_{l}} \widetilde{L}_{\eta_{l}} \quad \text { for each } \quad k=1,2, \ldots
$$

Thus $\left\{\widetilde{Y}_{k ; k=1, \ldots . . .}\right.$ is a decreasing sequence of continua, any one of which consists of $t^{k}$ triangles $\widetilde{T}_{v_{k}}$ and all joining them completive segments of orders $0,1, \ldots, k-1$.

Note that completive segments are all disjoint. Indeed, the 3 completive segments of order 0 (i.e. components of $\widetilde{L}$ ) are disjoint. Moreover, any one of them meets the triangles $\widetilde{T}_{0}, \widetilde{T}_{1}, \widetilde{T}_{2}$ and $\widetilde{T}_{3}$ in their vertices

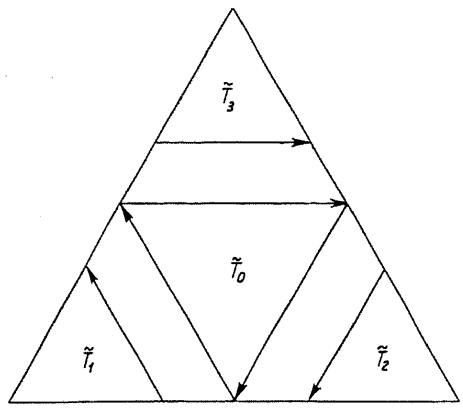

Fig. 2

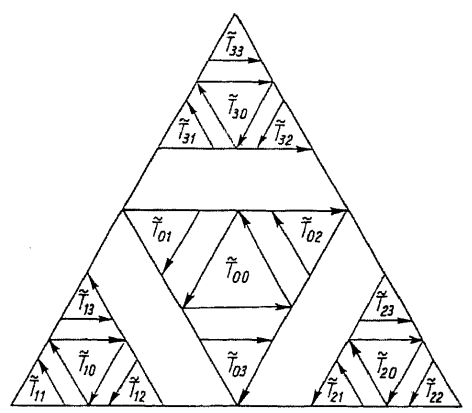

Fig. 3 only, and the completive segments of order 1 (i.e. components of $\bigcup_{\nu_{1} \in N_{1}} \widetilde{L_{v_{1}}}$ ) are the middle parts of the sides of these triangles. So they are disjoint one with another and with $\widetilde{L}$. The induction is obvious.

Notice also that because the operation $\gamma$ has the obvious property that the diameter $\delta$ of any of the 4 triangles $\widetilde{T}_{\boldsymbol{v}_{k^{0}}}, \widetilde{T}_{\boldsymbol{v}_{k^{1}}}, \widetilde{T}_{\boldsymbol{v}_{k^{2}}}, \widetilde{T}_{\boldsymbol{y}_{k^{3}}}$ is not greater than the half of the diameter of the instant triangle $\widetilde{T}_{v_{k}}$, then taking into account $\delta(\widetilde{T})=1$ we easily get

$$
\delta\left(\widetilde{T}_{v_{k}}\right) \leqslant 1 / 2^{k} \quad \text { for any } \quad v_{k} \in N_{k} \text { and } k=1,2, \ldots
$$

The next ste $p$ of our construction consists in adding up to any continuum $\widetilde{Y}_{k}$ some number of infinite sequences of segments in order to form a new decreasing sequence of larger continua $\left\{\widetilde{X}_{k}\right\}_{k=1,2, \ldots}$. This is a decisive step of our construction, because announced at the beginning continuum $\widetilde{X}$ will be the common part of all these continua $\widetilde{X}_{k}$, and the segments enlarging $\widetilde{Y}_{k}$ to $\widetilde{X}_{k}$ together with the completive ones will form the only non-degenerate elements of the decomposition of $\widetilde{X}$. Hence the whole rest of the paper depends on the manner of enlarging the continuum $\widetilde{Y}_{k}$ but it is, unfortunately, rather complicated.

We begin at an orientation of the sides of the triangles $\widetilde{T}_{v_{k}}$ of the continuum $\widetilde{Y}_{k}$. Namely, we shall assign the orientation to some (not 
to all) sides of the triangles $\widetilde{T}_{v_{k}}$, which will satisfy two following conditions:

(6) any two conjugate sides (see p. 27) will be oriented and the orientations will be the same,

( 7 ) no one vertex of any triangle $\widetilde{T}_{\nu_{k}}$ can be simultaneously initial and final point of its two oriented sides.

This orientation will be called consistent.

Since any side of the triangle $\widetilde{T}_{v_{k-1}}$ is conjugate with some side of a triangle $\widetilde{T}_{v_{k-1} i}$, where $i=1,2$ or 3 , then by (6) we have

(8) all 3 sides of any central triangle $\widetilde{T}_{v_{k-1}}$, where $v_{k-1} 0 \in N_{k}$ are oriented, and

(9) at least one side of any triangle $\widetilde{T}_{v_{k}}$, where $v_{k} \in N_{k}$, is oriented.

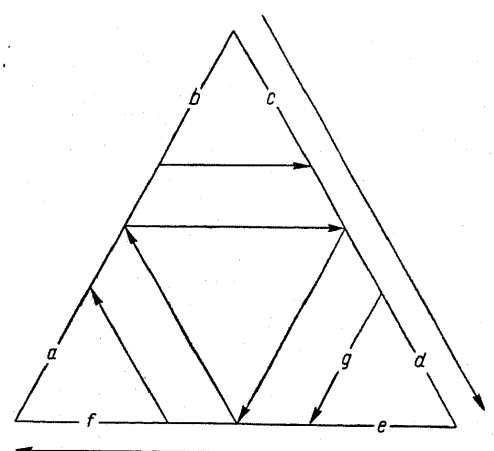

Fig. 4

The definition of the consistent orientation is inductive and starts with 4 triangles of $\widetilde{Y}_{1}$ : orient clockwise 3 sides of the triangle $\widetilde{T}_{0}$ and next orient, accordingly to (6), 3 sides conjugate with the just oriented (see Fig. 2, where the orientation is worked by arrows). The remaining 6 sides will be not oriented.

Suppose now that we have defined the consistent orientation of the sides of the triangles of $\widetilde{Y}_{k}$. Having in memory that from any triangle $\widetilde{T}_{\boldsymbol{v}_{k}}$ of $\widetilde{Y}_{k}$ are formed 4 triangles $\widetilde{T}_{\boldsymbol{v}^{0}}, \widetilde{T}_{\boldsymbol{v}^{1}}, \widetilde{T}_{\boldsymbol{v}_{\boldsymbol{k}^{2}}}, \widetilde{T}_{\boldsymbol{v}_{k^{3}}}$ of $\widetilde{Y}_{k+1}$, let us orient first by the following formulae these 6 sides of triangles $\widetilde{T}_{v_{k} 1}, \widetilde{T}_{v^{2}}$ and $\widetilde{T}_{\boldsymbol{v}_{k} 3}$, which lie on the sides of the triangle $\widetilde{T}_{v_{k}}$ (see Fig. 4 , where the exterior arrows show the orientation of sides of a triangle of $\widetilde{Y}_{k}$ and the arrows lying on the sides of smaller triangles of $\widetilde{Y}_{k+1}$ show their resulting orientation):

(i) if the side of the triangle $\widetilde{T}_{v_{k} i}$, where $i=1,2$ or 3 , is a part of a not oriented side of the triangle $\widetilde{T}_{v_{k}}$, then it remains not oriented itself (e.g. sides $a$ and $b$ on Fig. 4);

(ii) if the side of the triangle $\widetilde{T}_{v_{k} i}$, where $i=1,2$ or 3 , is a part of an oriented side of the triangle $\widetilde{T}_{v_{k}}$ but does not contain the final point of this oriented side, then it is also not oriented (e.g. sides $c$ and $e$ on Fig. 4);

(iii) if the side of the triangle $\widetilde{T}_{v_{k}}$, where $i=1,2$ or 3 , is a part of an oriented side of the triangle $\widetilde{T}_{v_{k}}$ and does contain the final point of this oriented side, then it inherits the orientation of this oriented side (e.g. sides $d$ and $f$ on Fig. 4).

Now let us turn to the 6 remaining sides of the 4 triangles $\widetilde{T}_{v_{k} 0}$, $\widetilde{T}_{y_{k} k^{2}}, \widetilde{T}_{v_{k^{2}}}$ and $\widetilde{T}_{v_{k} k^{3}}$, that is to those sides, which are not parts of a boundary of $\widetilde{T}_{v_{k}}$. They form 3 pairs of conjugate sides and therefore by virtue of (6) must be oriented.

To this effect we shall use one of the just oriented sides of these triangles, for in view of (9) and formula (iii) above at least one side of one of them is already oriented. The orientation of this side implies the agreeing with (7) orientation of the third side, i.e. of the conjugate one, of this triangle (e.g., in Fig. 4 the orientation of $d$ implies that of $g$ ), and therefore we can easily extend, in full accordance to (6) and (7), the orientation of this third side to the conjugate side of the triangle $\widetilde{T}_{v_{k}}{ }^{\circ}$ and next to the 2 other conjugate pairs of sides (see Fig. 4). Since orientation in $\widetilde{Y}_{k}$ was consistent, then it is obvious that this last part of our definition does not depend from which oriented side of $\widetilde{T}_{v_{k^{1}}}, \widetilde{T}_{v_{k^{2}}},{\widetilde{T_{y}}}_{\boldsymbol{k}^{3}}$ we start and, moreover, that defined in such a way orientation of the sides of all the triangles $\widetilde{T}_{\boldsymbol{v}_{k+1}}$, where $\boldsymbol{v}_{k+1} \in N_{k+1}$, is consistent. The induction is completed.

Having defined consistent orientation we may now construct in each trapesoid $\widetilde{P}_{v_{k}}^{i}$, where $v_{k} \in N_{k}, k=0,1, \ldots$ and $i=1,2,3$ (recall that index $v_{0}$ we simply omit: trapesoids $\widetilde{P}^{1}, \widetilde{P}^{2}$ and $\widetilde{P}^{3}$ complete $\widetilde{Y}_{1}$ to the triangle $\widetilde{T})$, a sequence of segments $\left\{v_{p_{k}}^{i},\right\}_{j=1,2, \ldots}$. The sides of $\widetilde{P}_{v_{k}}^{i}$ common with the triangles $\widetilde{T}_{y_{i} i}$ and $\widetilde{T}_{y_{k} 0}$ are, by the definition (see p. 27 ), conjugate and in view of $(6)$ both these sides are equally oriented.

Taking into account this orientation, let us denote (see Fig. 5) by $a_{1}^{a}$ the initial point and by $a_{\omega}^{\alpha}$ the final point of the side of $\widetilde{P}_{v_{k}}^{i}$ common with the triangle $\widetilde{T}_{v^{a}}(a=0, i)$. 
Observe now as changes the position of $\widetilde{P}_{v_{b}}^{i}$ when we pass from the continuum $\widetilde{Y}_{k+1}$ (the parts of which are triangles $\widetilde{T}_{v_{k} i}$ and $\widetilde{T}_{v_{k} 0}$ ) to $\widetilde{Y}_{k+2}$. Both triangles $\widetilde{T}_{y_{1} 0}$ and $\widetilde{T}_{y_{i}}$. are divided (in view of $\gamma$-operation), each into 4 smaller triangles and 3 trapesoids, the way that each oriented side of $\widetilde{P}_{v_{s}}^{i}$ is divided into 3 equal parts; the first and the third are sides of the new triangles of $\widetilde{Y}_{k+2}$, and the middle is a union of 2

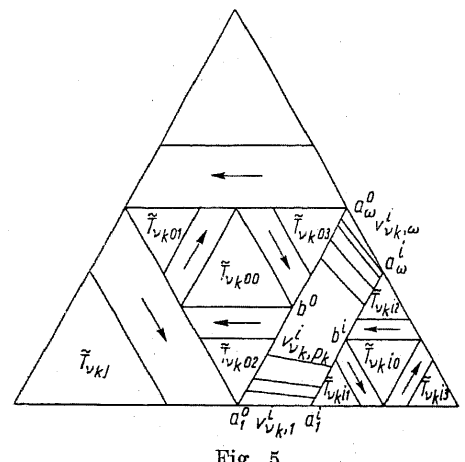

Fig. 5

sides of these 3 trapesoids. This middle segment-recall that it is a member of our decomposition-joins the vertices of 3 triangles of $\widetilde{Y}_{k+2}$. Passing now to $\widetilde{Y}_{k+3}$ we realise that the sides of the triangles of $\widetilde{Y}_{k+1}$ lying on oriented sides of $\widetilde{P}_{v_{k}}^{i}$ are divided again into 3 parts: extreme are sides of new triangles of $\widetilde{Y}_{k+1}$ and the middle are the members of our decomposition. All these middle segments (we called them completive) range out the Cantor ternary sets on oriented sides of $\widetilde{P}_{,}^{i}$. It is far to much we need; for our purpose will be enough that both points $a_{1}^{a}$ and $a_{\omega}^{a}$ are cluster points of the set $\overline{a_{1}^{a} a_{\omega}^{a}}-\bigcup_{l=0}^{\infty} \bigcup_{v_{l} \in N_{l}} \widetilde{L}_{p_{l}}$, which is obviously disjoint with the all completive segments.

Let $p_{k}$ be the $k$ th prime number. Denoting by $b^{a}$ the middle point of the segment $\overline{a_{1}^{a} a_{\omega}^{\alpha}}$ (i.e. the vertex of the triangle $T_{v_{k} a 0}$ lying on that segment), let us choose from the set $\overline{a_{1}^{a} a_{\omega}^{a}}-\bigcup_{l=0}^{\infty} \bigcup_{v_{l} \in N_{l}} \widetilde{L}_{p_{l}}$ the sequence of points $\left\{a_{j}^{\alpha}\right\}_{j=2,3, \ldots .}$ in such a way that $\left({ }^{8}\right)$

(8) It is a point that we lose the uniqueness of $X$. Roughly speaking, there are many sequences $\left\{a_{i}^{\alpha}\right\}$ satisfying conditions $(10)-(12)$ and for some of them the obtained continua are not bomeomorphic.
(10) $a_{j}^{\alpha}$ precedes $a_{j+1}^{a}$ (in the orientation of the side $\overline{a_{1}^{a} a_{\omega}^{\alpha}}$,

(11) $a_{p_{k}}^{a}$ precedes $b^{\alpha}$ (in the same orientation),

$$
\lim _{j \rightarrow \infty} a_{j}^{\alpha}=a_{\omega}^{\alpha}
$$

and join them pairwise, $a_{j}^{0}$ to $a_{j}^{i}$, by a segment which denote by $\tilde{v}_{v_{k}, j}^{i}$ $(i=1,2, \ldots, \omega)$. All segments $\tilde{v}_{\psi_{k}, j}^{i}$, where $v_{k} \in N_{k}, i=1,2,3$ and $j=2,3, \ldots$, we shall call enlarging continuum $\widetilde{Y}_{k}$ to continuum $\widetilde{X}_{k}$, which is to be defined now (the segments $\tilde{v}_{\boldsymbol{v}_{k}, 1}^{i}$ and $\tilde{v}_{\boldsymbol{v}_{k}, \omega}^{i}$ are not called entarging, as they are the subsets of $L_{v_{k}}$; in fact, $\left.\widetilde{L}_{v_{k}}=\bigcup_{i=1}^{3}\left(\tilde{v}_{v_{i}, i}^{i} \cup \tilde{v}_{v_{i}, \omega}^{i}\right)\right)$.

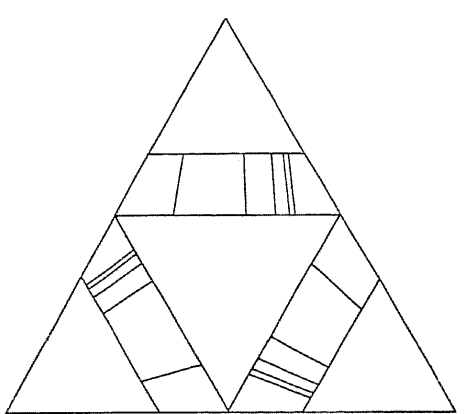

Fig. 6

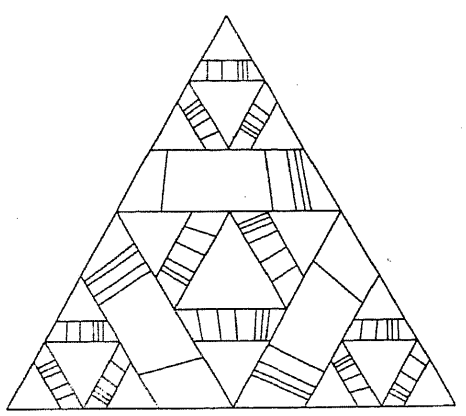

Fig. 7
Since by (12) the segments $\tilde{v}_{\boldsymbol{y}_{k}, j}^{i}$, where $j=2,3, \ldots$, form a conrergent sequence and $\operatorname{Lim}_{j} \tilde{v}_{v_{k}, j}^{i}=\tilde{v}_{\boldsymbol{v}_{k}, \omega}^{i}$ is a part of $\widetilde{L}_{v_{k}}$, then $\widetilde{X}_{1}$ (see Fig. 6) defined by adding to $\widetilde{Y}_{1}$ three sequences $\left\{\tilde{v}_{j}^{i}\right\}_{j=2,3, \ldots . . .}$, where $i=1,2,3$, is a continuum, $\widetilde{X}_{2}$ (see Fig. 7 ) defined by adding to $\widetilde{X}_{1} 4 \cdot 3$ sequences $\left\{v_{v_{k}, j}^{i}\right\} j=2,3, \ldots$, where $\nu_{1} \in N_{1}$ and $i=1,2,3$, is also a continuum, and generally, $\widetilde{X}_{k+1}$ defined for all $k=1,2, \ldots$ by the formula

$$
\widetilde{X}_{k+1}=\widetilde{X}_{k} \cup \bigcup_{y_{k} \in N_{k}} \bigcup_{i=1}^{3} \bigcup_{j=2}^{8} \tilde{v}_{y_{k}, j}^{i}
$$

is a continuum too. From that formula we infer, in particular, that $\widetilde{X}_{k+1} \subset \widetilde{X}_{k}$ and therefore $\widetilde{X}$, the common part of all $\widetilde{X}_{k}$,

$$
\widetilde{X}=\bigcap_{k=1}^{\infty} \widetilde{X}_{k}
$$

is a continuum as well.

Fundamer.la Mathematicae, T. LVII 
Continuum $\widetilde{X}$ is, by virtue of (4), (13) and (14), the union of a perfect set $\bigcap_{k=1}^{\infty} \bigcup_{v_{k} \in N_{k}} \widetilde{T}_{v_{k}}$, all completive segments and all segments enlarging $\widetilde{Y}_{k}$ to $\tilde{X}_{k}$ for $k=1,2, \ldots$

At last we come to the transformation of $\tilde{X}$ onto continuum $X$. This will be done with the help of an upper semicontinuous decomposition of $\widetilde{T}$.

The definition of the decomposition of $\widetilde{T}$ is simple: its only nondegenerate elements are the completive segments and the segments enlarging $\widetilde{Y}_{k}$ to $\widetilde{X}_{k}$ for all $k=1,2, \ldots$ We show that this decomposition is upper semicontinuous.

Indeed, the completive segments, as we stated before (see p. 29), are pairwise disjoint and by an easy induction starting from the segments enlarging $\widetilde{Y}_{1}$ to $\widetilde{X}_{1}$ we conclude that the segments enlarging $\widetilde{Y}_{k}$ to $\widetilde{X}_{k}$ for $k=1,2, \ldots$ are pairwise disjoint as well (cf. (10)). Moreover, in consequence of our cautious choosing of the points $a_{v_{k} i, j}^{\alpha}$ outside the completive segments, no one completive segment meets enlarging one. Hence all elements of our decomposition of $\widetilde{X}$ are disjoint and therefore it remains to show that the limit of any convergent sequence of these elements is contained in some other element. For this purpose recall that $\widetilde{L}_{v_{k}} \subset \widetilde{T}_{v_{k}}$ by the definition and $\delta\left(\widetilde{T}_{v_{k}}\right)<1 / 2^{k}$ by $(5)$, which means that for any $\varepsilon>0$ there exists only a finite number of completive segments of diameters greater than $\varepsilon>0$. Similarly in view of $\tilde{v}_{v_{k}, j}^{i} \subset \widetilde{P}_{v_{k}}^{i} \subset \widetilde{T}_{v_{k}}$ we have for the same $\varepsilon>0$ only a finite number of sequences of segments $\left\{\tilde{v}_{\boldsymbol{y}_{k}, j}^{i}\right\}_{j=2,3, \ldots}$, infinitely many of which have diameters greater than $\varepsilon$. It follows then that any converging sequence of elements of our decomposition, the limit of which does not reduce to a single point (and certainly only such sequences are here worth considering) is, excluding perhaps finitely many initial elements which is not of the slightest consequence here, a subsequence of some $\left\{\tilde{v}_{v_{k}, j}^{i}\right\}_{j=2,3, \ldots}$. But $\operatorname{Lim} \tilde{v}_{v_{k}, j}^{i}$ is, as we noted before (see p. 33), a part of one of the completive segments belonging to the set $\widetilde{L}_{v_{k}}$, i.e. a part of the element of our decomposition.

Hence our decomposition is upper semicontinuous and therefore, in view of Alexandroff's theorem (cf. [4], p. 42), there exists a continuous function $f$ running over $\widetilde{T}$, the counter-images of which are the elements of our decomposition. The hyperspace of our decomposition is then equal to $f(\widetilde{T})$. Since the only elements of our decomposition of $\widetilde{T}$ are segments and points, otherwise continua not separating the plane, hence by virtue of Moore's theorem (cf. [4], p. 380) the hyperspace $f\left(\widetilde{T^{\prime}}\right)$ is a plane continuum as well. (The reader can imagine $f(\widetilde{T})$ as a continuum obtaining by contracting to a point any element of our decomposition.)
Let us accept the simple convention that the images under the function $f$ of the triangles $\widetilde{T}_{v_{k}}$, trapesoids $\widetilde{P}_{v_{k}}^{i}$, continua $\widetilde{X}_{k}$ and $\widetilde{X}$, and segments $\tilde{v}_{v_{k}, j}^{i}$ we shall denote simply by dropping the wavy line. We shall then write $T_{\boldsymbol{v}_{k}}$ instead of $f\left(\widetilde{T}_{\boldsymbol{v}_{k}}\right)$, etc.

By (4) and (13) we have

$$
\widetilde{X}_{k}=\bigcup_{v_{k} \in N_{k}} \widetilde{T}_{v_{k}} \cup\left\{\bigcup_{l=0}^{k-1} \bigcup_{v_{l} \in N_{l}}\left[\widetilde{L}_{v_{l}} \cup \bigcup_{i=1}^{3} \bigcup_{j=2}^{\infty} \tilde{v}_{v_{l}, j}^{i}\right]\right\} \text {. }
$$

Since $\widetilde{X}_{k}$ is continum and all the elements in $\{\ldots\}$ are disjoint segments, so any one of them meets $\bigcup_{v_{k} \in N_{k}} \widetilde{T}_{v_{k}}$, and therefore

$$
X_{k}=\bigcup_{v_{k} \in N_{k}} T_{v_{k}}
$$

(see Figs. 8 and 9 , where $\widetilde{X}_{1}$ and $\widetilde{X}_{2}$ are shown).

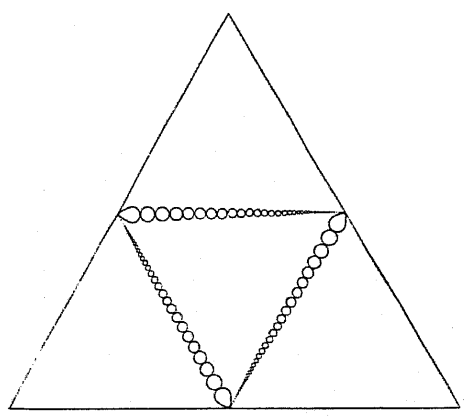

Fig. 8

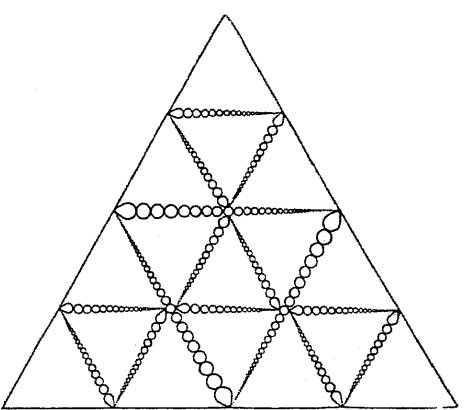

Fig. 9
Note by the way that since the triangle $\widetilde{T}_{v_{c}}$ is a topological dise and the elements of our decomposition restricted to $\widetilde{T}_{v_{k}}$ are, as in $\widetilde{T}$, the points and segments only, then by the quoted above Moore's theorem the continuum $T_{y_{k}}$ is also a topological disc. For that reason we shall continue to call it triangle. The vertices of $T_{v_{k}}$ are the images under the function $f$ of the vertices of $\widetilde{T}_{v_{k}}$. Since the decomposition restricted to a side $\overline{a b}$ of the triangle $\widetilde{T}_{\nu_{k}}$ consists of points and segments only, then $a b$ is transformed by $f$ onto an arc, whose ends are $f(a)$ and $f(b)$. We shall call this arc (together with its end-points) the side of $T_{v_{e}}$. The boundary of the triangle $T_{y_{k}}$ consists then of the 3 just defined sides meeting in the just defined vertices. We also preserve the orientation of the sides of the triangles $T_{v_{k}}$ (the side of ends $f(a)$ and $f(b)$ of the 
triangle $T_{y_{k}}$ is oriented from $f(a)$ to $f(b)$ if and only if the side $\overline{a b}$ of the triangle $\widetilde{T}_{v_{k}}$ is oriented from $a$ to $b$ ) and the name of conjugate sides (two sides of triangles $T_{v_{k}{ }^{0}}$ and $T_{\boldsymbol{v}_{k} i}$ are conjugate if and only if they are the images under $f$ of two conjugate sides of triangles $\widetilde{T}_{v_{k^{0}}}$ and $\widetilde{T}_{\boldsymbol{v}_{i}}$ ). One does not need to add that the inherited in such a way orientation is consistent and satisfies (6)-(9).

In view of $\widetilde{X}_{k+1} \subset \widetilde{X}_{k}$ we have obviously

$$
X_{k+1} \subset X_{k}
$$

whence by $(14)\left({ }^{9}\right)$

Finally, putting

$$
X=\bigcap_{k=1}^{\infty} X_{k}
$$

$$
\varepsilon_{k}=\sup _{v_{k} \in N_{k}} \delta\left(T_{\psi_{k}}\right)
$$

we hare by (5) and the continuity of $f$

$$
\lim _{k \rightarrow \infty} \varepsilon_{k}=0 \text {. }
$$

§ 3. Main geometrical features of the construction. Let us turn back for a moment to the trapesoid $\widetilde{P}_{\boldsymbol{v}_{k}}^{i}$ (see Fig. 5). Since the only non-degenerate elements of our decomposition restricted to $\widetilde{P}_{\boldsymbol{v}_{k}}^{i}$ are segments $\tilde{v}_{v_{k}, j}^{i}(j=1,2, \ldots, \omega)$ and completive segments lying on its 2 oriented sides, then obviously its hyperspace $P_{\boldsymbol{v}_{k}}^{i}\left(=f\left(\widetilde{P}_{\boldsymbol{v}_{k}}^{i}\right)\right.$ by the definition of $f$ ) is a sequence of topological dises joined by the points $v_{v_{k}, i}^{i}$ (see Fig. 10).

Let us call $P_{v_{k}}^{i}$ a chaplet and denote composing it dises consecutively by $Q_{v_{k}, j}^{i}(j=1,2, \ldots)$. Let us call also $v_{v_{k}, j}^{i}$ for $j=2,3, \ldots$ joints, $v_{v_{k}, 1}^{i}$ initial and $v_{v_{k}, \omega}^{i}$ final points of $P_{v_{k}}^{i}$. The last 2 points we shall also call shortly extreme points of $P_{v_{k}}^{i}$. We shall say that chaplet $P_{v_{k}}^{i}$.divorces triangles $T_{v_{k^{0}} 0}$ and $T_{\boldsymbol{v}_{k} i}$ of $X_{k+1}$.

(9) We apply here the following simple property of the continuous mappings: If $\left\{A_{k}\right\}$ is a decreasing sequence of compact sets and $Y$ is a topological space, then $f\left(\cap A_{k}\right)=\cap f\left(A_{k}\right)$ for every continuous function $f: A_{1} \rightarrow Y$.

In fact, the left side is contained in the right one without any hypotheses concerning neither sets nor function (see [3], p. 17) and if $b \in \cap f\left(A_{k}\right)$, then there exists a sequence $\left\{a_{k}\right\}$ such that $a_{k} \in A_{k}$ and $f\left(a_{k}\right)=b$ for $k=1,2, \ldots$ Removing now, if necessary, some terms of the sequence $\left\{A_{k}\right\}$, we may assume that the sequence of points $\left\{a_{k}\right\}$ is converging. Putting $a=\lim a_{k}$ we have $f(a)=b$ by the continuity of $f$. But the sets $A_{k}$ are compact and form a decreasing sequence. Hence we have also $a \in \cap A_{k}$ and therefore $b \in f\left(\cap A_{k}\right)$.
Examine now the situation of $P_{\boldsymbol{v}_{k}}^{i}$ in $X_{k+2}$. Any one of both triangles $T_{v_{k^{\alpha}}}(a=0, i)$ is divided into 4 smaller triangles $T_{v_{k} a j}(j=0,1,2,3)$ in the way that 2 of them have whole sides common with $P_{\boldsymbol{v}_{k}}^{i}$ and the central triangle $T_{v_{k} a 0}$ common vertex only. In view of (11) this vertex lies after the joint $v_{\boldsymbol{v}_{k}, p_{k}}^{i}$ (in the orientation from $v_{\boldsymbol{v}_{k}, 1}^{i}$ to $v_{\boldsymbol{v}_{k}, \omega}^{\boldsymbol{i}}$ ) and therefore

(20) the vertex of $T_{\boldsymbol{v}_{k} 00}^{i}(\alpha=0$ or $i)$ lying on a chaplet $P_{\boldsymbol{v}_{k}}^{i}$ does not belong to the union $\bigcup_{j=1}^{p_{k}-1} Q_{\boldsymbol{v}_{k}, j}^{i}$ of first $p_{k}-1$ dises of $P_{\boldsymbol{v}_{k}}^{i}$.

This is a crucial property of the construction. It will be used in the proof of decomposition (2) only and an application of it, however often, is stereotypical. Roughly speaking, we shall consider starting from a point $v_{\boldsymbol{v}_{k}, 1}^{i}$ a connected union consisting of first $p_{k}-1$ discs of a chaplet $P_{\boldsymbol{v}_{k}}^{i}$, to which we add first $p_{k}-1$ discs of all chaplets, the initials of which belong to them, and so on. Property (20) assures that

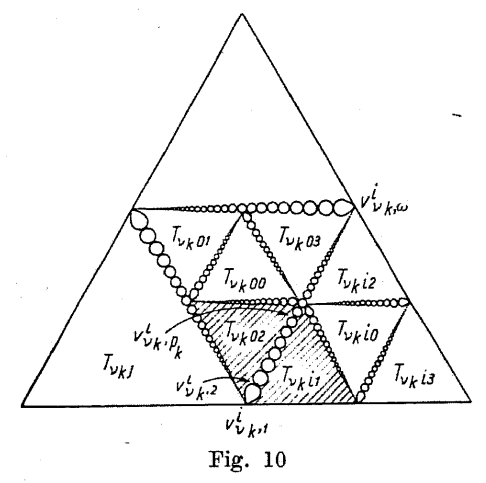

this union does not contain discs of a chaplet, the initial of which is vertex of $T_{v_{z^{0}} 0}$ : actually, this union is contained in 2 shadowed in Fig. 10 triangles. This fact has some important consequences.

Now look at $X_{1}$ (see Fig. 8). Topologically, it is a triangle $T$, in which 3 points, one in each side, are joined by 3 pairwise disjoint and lying in interior of $T$ (except of their extreme points) chaplets $P^{1}, P^{2}$ and $P^{3}$ in the way that each extreme point is simultaneously initial and final of 2 chaplets meeting in it. We get $X_{1}$ by removing interior of these chaplets. So $X_{1}$ consists of 4 topological triangles $T_{0}, T_{1}, T_{2}$ and $T_{3}$. By the definition of the orientation (see pp. 29-31 and 35-36) their only oriented sides are those lying on chaplets $P^{i}$. More precisely, the side of ends $a$ 
and $b$ of triangle $T_{i}$ is oriented from $a$ to $b$ if and only if it contains all points $\left\{v_{j}^{i}\right\}_{j=1,2, \ldots}$ and $b=v_{\omega}^{i}$ for some $i=1,2$ or 3 .

Continuum $X_{2}$ (see Fig. 9) can be obtained from $X_{1}$ by the application of the similar procedure to each of the 4 topological triangles $T_{i}$ $(i=0,1,2,3)$. As previously, 3 points (one in each side) of each triangle $T_{i}$ are joined by 3 pairwise disjoint and lying in interior of $T_{i}$ (except of their extreme points) chaplets $P_{i}^{1}, P_{i}^{2}, P_{i}^{3}$ in the way that each extreme point is simultaneously initial and final of 2 chaplets meeting in it. As previously, we get $X_{2}$ by removing interiors of these 4.3 chaplets. However, these 12 chaplets satisfy 2 essential additional conditions: (20) explained above and that of consistent orientation. (20) means that the vertex of $T_{\alpha 0}\left(\alpha=0\right.$ or $i$ ) lying on $P^{i}$ does not belong to $Q_{1}^{i}$. Moreover, this vertex is not a joint of chaplet $P^{i}$ and therefore is a common vertex of 3 only triangles of $X_{2}$ (those lying in $T_{\alpha}$ ). And consistent orientation means that if we consider the orientation of sides of triangles $T_{v_{2}}$ of $X_{2}$ as defined on pp. 29-31 and 35-36, then it satisfies (7). Notice that side of ends $a$ and $b$ of triangle $T_{y_{2}}$ is oriented from $a$ to $b$ if and only if it contains almost all points of some sequence of joints and $b$ is the final point of this sequence.

By the application of the same procedure (i.e. preserving (20) and consistent orientation) to each of $4^{2}$ triangles $T_{p_{2}}$ of $X_{2}$ continuum $X_{3}$ can be obtained, and applying it in the same way to each of $4^{3}$ triangles $T_{r_{3}}$ of $X_{3}$ continuum $X_{4}$ can be obtained, and so on.

By an easy inductive argument, simply checking the definition of consistent orientation, we find out that:

(21) the side of ends $a$ and $b$ of triangle $T_{v_{k}}$ is oriented from $a$ to $b$ if and only if it contains almost all points of some sequence of joints and $b$ is the final point of this sequence.

The procedure described above allows us to construct a decreasing sequence $\left\{X_{k}\right\}_{k=1,2, \ldots}$ of continua $X_{k}$ determinating $X$ by formula (17). It remains, however, one condition concerning this whole sequence. Namely, we must secure that diameters of triangles $T_{v_{k}}$ of $X_{k}$ tend to zero together with $k \rightarrow \infty$ (cf. (18) and (19)). And just for that reason, in view of serious technical defficulties connected with correspondingly skillful inscription of new chaplets, we had to betake ourselves to less intuitive but in this case far more simple apparatus of upper semicontinuous decompositions. Nevertheless, I hope the very idea of the con-
struction is clear.

In $\S 5$ some more important properties of the construction will be explicitly noted and those less obvious will be provided in a proof. But that is all. In order to make this paper readable I consciously resign from taking down all the properties of the construction, upon which the further proofs will be based. For a reader who understands the idea, given explanations will be more than enough. It seems to me hopeless and useless to write a huge paper containing all details, logically correct and step by step verifiable, but at the same time completely unreadable.

§ 4. Proof that $X$ is hereditarily locally connected. Let us recall that a continuum $C$ contained in a space $Y$ is calleda continbuum of convergence of $Y$ provided that there exists a sequence of pairwise disjoint continua $C_{i} \subset Y$ such that $C=\operatorname{Lim} C_{i}$ and $C \cap C_{i}=0$ for all $i$ (see [4], p. 1i6).

Urysohn and Zarankiewicz (see [4], p. 196) have proved that contimuum $Y$ is hereditarily locally connected if and only if each continuum of convergence of $Y$ reduces to a point, or, in other words, if it does not contain any proper continuum of cergence.

4.1. Among each three different triangles of $X_{k}$ there are two such that their common part is empty or consists of one point only.

Proof is by an easy induction. For $k=1$ it is clearly obvious, because each pair of non-central triangles of $X_{1}$ has one point common only (see Fig. 8). Suppose that it is true for $k \geqslant 1$ and take three different triangles of $X_{k+1}$. If they are subtriangles of the same triangle of $X_{k}$, then it is equally obvious as for $k=1$, and if each of them lies in another triangle of $X_{k}$, then it follows by induction hypothesis. So remains the case that 2 of them lie in one triangle of $X_{k}$ and the third in another. If both these 2 are not central, then they meet in one point only, and if one of them is central, then it has only one of its vertices common with each side of that triangle of $X_{k}$ in which it lies, and, consequently, can have only that vertex common with the third.

4.2. If $C$ is a continuum of convergence of $X_{k}$, then $C$ is contained in a union of 2 triangles of $X_{k}$.

Proof. Let $C=\operatorname{Lim} C_{i}$, where $C_{i} \cap C_{j}=0$ and $C \cap C_{i}=0$ for $i, j=1,2, \ldots$ and $i \neq j$, and suppose, to the contrary, that $C$ meets 3 different triangles $T_{v_{k}}, T_{\mu_{k}}, T_{\pi_{k}}$ in their interiors ${ }^{\circ} T_{v_{k}},{ }^{0} T_{\mu_{k}},{ }^{\circ} T_{\pi_{k}}$ with regard to $X_{k}$. Applying 4.1, let

(a) $\quad T_{\nu_{k}} \cap T_{\mu_{k}}$ be empty or consists of one point only.

Choosing for each $i=1,2, \ldots$ a pair of points

$$
a_{i} \in C_{i} \cap{ }^{0} T_{v_{k}} \text { and } b_{i} \in C_{i} \cap{ }^{0} T_{\mu_{k}} \text {, }
$$

we shall show that there exists a sequence of pairwise disjoint arcs $L_{i} \subset X_{k}$ of ends $a_{i}$ and $b_{i}$.

Indeed, $X_{k}$ is, as a union of finitely many topological discs $T_{\tau_{k}}$, locally connected. Let $K(A, \eta)$ be, for any $A \subset X_{k}$ and $\eta>0$, the set of all points of $X_{k}$ which can be joined with $A$ by an are $L \subset X_{k}$ of dia- 
meter $\delta(L) \leqslant \eta$. Since any such $K(A, \eta)$ is a locally connected continuum, then each $K\left(C_{i}, \varepsilon_{i}\right)$, where

$$
3 \varepsilon_{i}=\inf _{\substack{x \in C_{i} \\ y \in \cup_{i \neq j} C_{i}}} \varrho(x, y),
$$

contains an arc $L_{i}$ of ends $a_{i}$ and $b_{i}$. Moreover, in view of (c) we have $K\left(C_{i}, \varepsilon_{i}\right) \cap K\left(C_{j}, \varepsilon_{j}\right)=0$ for $i \neq j$ and therefore all $L_{i}$ are pairwise disjoint.

In view of (a) and (b), infinitely many arcs are not contained in $T_{v_{k}} \cup T_{\mu_{k}}$, the interiors of which they meet, and therefore each arc $L_{i}$ must "pass" through some other triangle $T_{\tau_{t}}$, i.e. going along $L_{i}$ from $a_{i}$ to $b_{i}$ we "come in" some triangle $T_{\tau_{k}}$ through one of its sides and "come out" through another. Since there is only finitely many triangles

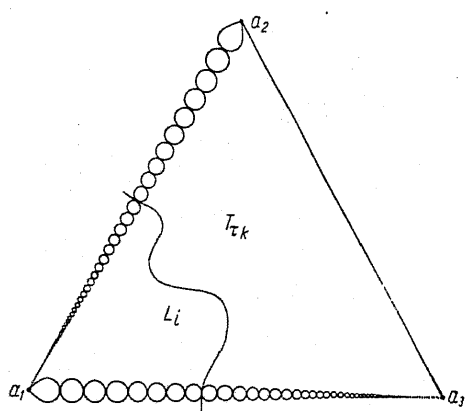

Fig. 11

$T_{v_{k}}$ of $X_{k}$, then we may suppose, removing if necessary some subsequences of $\left\{L_{i}\right\}_{i=1,2, \ldots}$, that all $L_{i}$ "pass" through the same $T_{\tau_{1}}$ and, moreover, that all "come in" through the same "front" side of $T_{\tau_{k}}$ and all "come out" through the same "back" side of $T_{\tau_{k}}$.

The "entering" points of $L_{i}$ belong, of course, to the boundary of $T_{\tau_{k}}$ with regard to $X_{k}$ and since all of them lie in the same side of $T_{\tau_{k}}$, then they are the points of some sequence $\left\{v_{\boldsymbol{v}_{m}, j}^{p}\right\}_{j=1,2, \ldots}$ and the limit $\boldsymbol{v}_{\boldsymbol{y}_{m, \infty}}^{\boldsymbol{p}}$ of this sequence is a vertex of $T_{\tau_{k}}$ (see Fig. 11). Denote this vertex by $a_{1}$, the second end of the "front" side by $a_{2}$, and the third vertex of $T_{\tau_{k}}$ by $a_{3}$. By $(21)$ the side $\left(a_{1} a_{2}\right)$ is then oriented from $a_{2}$ to $a_{1}$.

Now two cases are possible: the "back" side is $\left(a_{1} a_{3}\right)$ or $\left(a_{2} a_{3}\right)$. Since the argumentation is in both cases identical, consider only the first (see Fig. 11). The "coming out" points of $L_{i}$ are, as previously, the points of some (other) sequence $\left\{v_{y_{n}, j}^{s}\right\}_{j=1,2, \ldots}$ and the limit $v_{v_{n}, \omega}^{s}$ of this sequence is $a_{2}$ or $a_{3}$. But in view of $(21)$ the side $\left(a_{1} a_{3}\right)$ is, as containing infinitely many points of a sequence $\left\{v_{v_{n}, j}^{s}\right\}_{j=1,2, \ldots}$, oriented, and, in view of (7) and the orientation of the side $\left(a_{1} a_{2}\right), v_{p_{n}, \omega}^{s}=a_{3}$. It is a contradiction, because any arc on $T_{\tau_{k}}$ joining interior points of the sides $\left(a_{1} a_{2}\right)$ and $\left(a_{1} a_{3}\right)$ separates $T_{\tau_{k}}$ between infinite parts of sequences $\left\{v_{v_{m}, j}^{p}\right\}_{j=1,2, \ldots}$ and $\left\{v_{\boldsymbol{v}_{n}, j}^{s}\right\}_{j=1,2, \ldots}$ and therefore the $\operatorname{arcs} L_{i}$ cannot be disjoint.

It follows then that any continuum of convergence of $\bar{X}_{k}$ is contained in at least two triangles of $X_{k}$.

THEOREM 1. Continuum $X$ is hereditarily locally connected.

Proof. It suffices to show (see p. 39) that "if $C$ is a continuum of convergence of $X$, then $\delta(C)=0$. But if $C$ is a continuum of convergence of $X$, then we infer from inclusions $C \subset X \subset X_{k}$ following by (17) and hypothesis $C \subset X$, that $C$ is a continuum of convergence of each $X_{k}$. In view of 4.2 and $(18)$ we have then $\delta(C) \leqslant 2 \varepsilon_{k}$ for each $k=1,2, \ldots$, whence $\delta(C) \leqslant 0$ by $(19)$.

§ 5. Some simple properties of the construction. Before we proceed to the proof of the existence of the decomposability (2) of $X$ let us state some simple properties of our construction. Since they are often obvious, then only few of them will be provided in explanation or outline of the proof.

Let us observe first that

$$
\operatorname{Fr}\left(X_{k}\right)=\bigcup_{v_{k} \in N_{k}} \operatorname{Fr}\left(T_{v_{k}}\right)
$$

We shall often use the complementary sets of continua $X_{k}$. For that purpose notice that since $X_{k+1}$ arises from $X_{k}$ by a suitable inscription of 3 chaplets into each triangle $T_{v_{k}}$, then

and

(24) each $P_{v_{k}}^{i}$ meets $\overline{E^{2}-X_{k}}$ in its extreme points $v_{v_{k}, 1}^{i}$ and $v_{v_{k}, \omega}^{i}$ only.

It implies, in particular, that

$$
\operatorname{Fr}\left(X_{k+1}\right)=\operatorname{Fr}\left(X_{k}\right) \cup \bigcup_{\nu_{k} \in N_{k}} \bigcup_{i=1}^{3} \operatorname{Fr}\left(P_{p_{k}}^{i}\right)
$$

whence

$$
\operatorname{Fr}\left(X_{k}\right) \subset \operatorname{Fr}\left(X_{k+1}\right)
$$

Thus obviously $\operatorname{Fr}\left(X_{k}\right) \subset \operatorname{Fr}\left(X_{k+l}\right)$ for each $l=1,2, \ldots$ and since $\operatorname{Fr}\left(X_{k+l}\right) \subset X_{k+l}$, because $X_{k+l}$ is a continuum, then in view of (16) and 
(1i) we have $\operatorname{Fr}\left(X_{k}\right) \subset X$ for all $k=1,2, \ldots$ Hence and from (18), (19) and (22) we infer that

(27) $\bigcup_{k=1}^{\infty} \operatorname{Fr}\left(X_{k}\right)$ is simultaneously dense and boundary subset of $X$.

Also another important property of $\operatorname{Fr}\left(X_{k}\right)$ will be needed. Namely,

$$
\text { no point of } \operatorname{Fr}\left(X_{k}\right) \text { sepärates it. }
$$

Proof is inductive and starts with a simple closed curve $\operatorname{Fr}\left(X_{0}\right)$ as by (15) $X_{0}=T$. Assuming (28) for $\operatorname{Fr}\left(X_{k}\right)$, let us take a point $p \in \operatorname{Fr}\left(X_{k+1}\right)-\operatorname{Fr}\left(X_{k}\right)$. By virtue of (25) $p \in \operatorname{Fr}\left(P_{v_{k}}^{i}\right)$. Since $\operatorname{Fr}\left(P_{\boldsymbol{v}_{k}}^{i}\right)$ is a union of a sequence of simple closed curves $\operatorname{Fr}\left(Q_{v_{k}, j}^{i}\right)$ joined consecutively by joints $v_{v_{k}, j}^{i}$, and of a point $v_{v_{k}, \omega}^{i}$, then it can be separated by one of its joints only. But in such a case $\operatorname{Fr}\left(P_{v_{k}}^{i}\right)-(p)$ is a union of 2 connected sets, one of which contains $v_{\psi_{k}, 1}^{i}$ and the other $v_{v_{k, \omega}, \omega}^{i}$, and these 2 points are precisely the common points of $\operatorname{Fr}\left(P_{\boldsymbol{v}_{k}}^{i}\right)$,with $\operatorname{Fr}\left(X_{k}\right)$. Hence $\operatorname{Fr}\left(X_{k}\right) \cup \operatorname{Fr}\left(P_{\boldsymbol{v}_{k}}^{i}\right)-(p)$ is connected for each $p \in \operatorname{Fr}\left(P_{\boldsymbol{v}_{k}}^{i}\right)$ and it proves (28) for $k+1$.

From (27) and (28) we get at once

$$
\text { no point of } X \text { separates it. }
$$

In strong connexion with the above property is the following one (30)

$$
\text { If } A \text { is subset of } \operatorname{Fr}(T) \text {, then } X-A \text { is connected. }
$$

For the proof notice first that $\operatorname{Fr}\left(X_{2}\right)-\operatorname{Fr}(T)$ consists of 4 components: the boundaries of 3 chaplets of order 2 having both extreme points on $\operatorname{Fr}(T)$ and the rest (see Fig. 9). The rest forms $2 \varepsilon_{2}$-net for $X_{2}$ and therefore for $X$, because $X \subset X_{2}$ by (17). Now, the boundaries of chaplets of order 3 join these 3 exceptional chaplets of order 2 with the "rest" and therefore one of the components of $\operatorname{Fr}\left(X_{3}\right)-\operatorname{Fr}(T)$ contains the boundaries of all chaplets of order 3 except of those 3 (lying near the vertices of $T$ ) which have both extreme points on $\operatorname{Fr}(T)$. This component is $2 \varepsilon_{3}$-net for $X$. Proceeding by .induction, we get an increasing sequence of sets, each of which is a component of $\operatorname{Fr}\left(X_{k}\right)-\operatorname{Fr}(T)$ and $2 \varepsilon_{k}$-net for $X$. Its union is then a connected and dense subset of $X$. Property $(30)$ is a mere consequence of the last statement.

Properties (27), (28), (29) and (30) are valid for each triangle $T_{y_{k}}$ separately. Namely, using literarily the same argumentation (with the only restriction to $T_{v_{k}}$ instead of $T$ ) one proves that

(31) $\bigcup_{l=k}^{\infty} T_{y_{k}} \cap \operatorname{Fr}\left(X_{l}\right)$ is simultaneously dense and boundary subset of $T_{\boldsymbol{y}_{k}} \cap X$,
(32) no point of $T_{p_{\mathrm{k}}} \cap \operatorname{Fr}\left(X_{l}\right)$ separates it,

(33) no point of $T_{v_{k}} \cap X$ separates it,

(34) if $A$ is a subset of $\operatorname{Fr}\left(T_{\eta_{k}}\right)$, then $T_{v_{k}} \cap X-A$ is connected.

By an easy induction we also find that

(35) each side of triangle $T_{\nu_{k}}$ is a subset of a side of triangle $T$ or of one only chaplet of order $<k$;

(36) each vertex $b$ of each triangle $T_{v_{k}} \subset \operatorname{Int}(T)$ is initial or final point of a chaplet and this chaptet contains a side of $T_{v_{k}}$, one end of which is $b$;

(37) if $v_{\boldsymbol{v}_{k+p}, 1}^{i} \in \operatorname{Int}\left(T_{\boldsymbol{v}_{k}}\right)$, then $P_{\boldsymbol{v}_{k+p}, 1}^{i}-v_{\boldsymbol{v}_{k+p}, \omega}^{i} \subset \operatorname{Int}\left(T_{\boldsymbol{v}_{k}}\right)$,

(38) if $p \in \operatorname{Fr}\left(T_{v_{k}}\right)$, but $p$ is not a vertex of $T_{v_{k}}$, then for each $\varepsilon>0$ there exists a triangle $T_{v_{k+r}}$ of diameter $\delta\left(T_{v_{k+r}}\right)<\varepsilon$, which contains $p$ and has the following property: one of the ends of the side $T_{p_{b+3} \text { con- }}$ taining $p$ is an initial point and the other end a final point of chaplets, each of which contains one of 2 other sides of $T_{v_{k+r}}$ (look at the sides of $T_{v_{\mathrm{z}}}$ in Fig. 9).

(39) If continuum $M$ is contained in $P_{v_{k}}^{i}$ and contains at most one of extreme points of $P_{\boldsymbol{v}_{k}}^{i}$, then $X_{k+1}-M$ is connected.

Indeed, by its definition $P_{v_{k}}^{i}$ divorces 2 triangles $T_{v_{k} i}$ and $T_{\nu_{k}{ }^{0}}$ of $X_{k+1}$ and has only its extreme points common with $\operatorname{Fr}\left(X_{k}\right)$. Hence $T_{v_{k} i}-M, \quad T_{v_{k} 0}-M$ and $\operatorname{Fr}\left(X_{k}\right)-M$ are connected, and $T_{v_{k+1}} \cap M=0$ for $\nu_{k+1} \neq v_{k}^{i}$ or $v_{k 0}$. Joining then to $\operatorname{Fr}\left(X_{k}\right)-M$ the sets $T_{v_{k} i}-M$, $T_{v_{k} 0}-M$ and all other triangles $T_{v_{k+1}}$ we get a connected set $X_{k+1}-M$.

(40) If continuum $K$ separates the plane between 2 triangles of $X_{k}$ and $K \subset \overline{E^{2}-X_{k}}$, then $K$ contains all joints' of some chaplet of order $\leqslant k-1$.

Proof is inductive. For $k=1$ it is obvious.

Supposing then (40) for $k$, take a continuum $K$ contained in $\overline{E^{2}-X_{k+1}}$ and separating the plane between some points of $X_{k+1}$. We shall show that $K$ contains all joints of some chaplet of order $\leqslant k$.

For that purpose, let us start with considering common parts of $K$ with the chaplets of order $k$. Since each continuum lying in a chaplet and containing its extreme points must contain all its joints, then we may assume that no component $M$ of $K \cap P_{v_{k}}^{i}$, where $v_{k} \in N_{k}$ and $i=1,2,3$, contains simultaneously both extreme points of $P_{\boldsymbol{v}_{k}}^{i}$, and therefore, in view of (39), that $M$ does not separate $X_{k+1}$. Since $P_{y_{k}}^{i}$ has only its extreme points common with the rest of $\overline{E^{2}-X_{k+1}}$, then we may write $K=\overline{K-P_{v_{k}}^{i}} \cup M_{1} \cup M_{2}$, where $M_{1}$ and $M_{2}$ are disjoint com- 
ponents of $K \cap P_{\boldsymbol{y}_{k}}^{i}$ containing the initial and final points of $P_{\nu_{k}}^{i}$ respectively $\left(M_{1}\right.$ or $M_{2}$ may be empty), and $\overline{K-P_{\nu_{k}}^{i}}$ is obviously a continuum. Since neither $M_{1}$ nor $M_{2}$ separates the plane between points of $X_{k+1}$ and $K$ does, then, by First Theorem of Janiszewski (see [4], p. 355), $\overline{\boldsymbol{K}-P_{\boldsymbol{v}_{k}}^{i}}$ does as well. It means that removing from $K$ all its parts lying in $3 \cdot 4^{k}$ chaplets $P_{v_{k}}^{i}$ we get continuum $K^{\prime}$ contained in $\overline{E^{2}-\bar{X}_{k}}$ and still separating plane between some points of $X_{k+1}$. Since $X_{k+1} \subset X_{k}$, then to finish the proof we apply the induction hypothesis.

§ 6. Some preliminary lemmas. Let $V_{m}$ be the set of all those joints $v_{v_{k}, j}^{i}$, the consectuve number $j$ of which is a multiple of the $m$ th prime number $p_{m}$. Since no point is simultaneously a joint of 2 chaplets, then obviously

$$
V_{l} \cap V_{m}=0 \quad \text { for } \quad l, m=1,2, \ldots, \quad l \neq m .
$$

The union $\bigcup_{n=1}^{\infty} V_{m}$ forms the announced at the beginning (see p. 27) set $V$.

Denote also by $\nabla_{0}$ the set of initial points of all chaplets, i.e. the set consisting of all points $v_{v_{k}, 1}^{i}$.

From that time until the end of the paper let $n$ be a fixed naturat number.

Take a chaplet $P_{\boldsymbol{v}_{\boldsymbol{k}}}^{i}$ and consider its subset consisting of interiors of all forming it dises $Q_{v_{k}, j}^{i}(j=1,2, \ldots)$, of all its joints $v_{v_{k}, j}^{i}$ except those belonging to $V_{n}$ and, finally, of initial points of all other chaplets lying in the boundaries of dises $Q_{v_{k}, j}^{i}$. The components of this subset are then the unions of interiors of $n$ consecutive discs joined by joints lying between them, and of a set of initial points of other chaplets of all orders lying on boundaries of these dises. We shall call these components links and will denote them consecutively by $P_{\boldsymbol{v}_{k}, j}^{i}$, where $j=1,2, \ldots$

Especially important is the role in the sequel played by the first link $P_{v_{k}, 1}^{i}$. Obviously,

$$
\overline{P_{\boldsymbol{v}_{k}, 1}^{i}}=\bigcup_{j=1}^{p_{k}-1} Q_{v_{k}, j}^{i}
$$

The proof of the decomposition (2) of $X$ requires considering the set

$$
Z=(T-X) \cup \bigcup_{n \neq l=0}^{\infty} \nabla_{l}
$$

consisting of the interiors of all dises forming chaplets, all their initial points and all their joints except those, the consecutive number $j$ of which is a multiple of the $p_{n}$ th prime number. Since this set lies mainly in the complement of $X$ to $T$, then one seems to be easier to discover some of its important properties by considering it as far as it appears in the successive approximations $X_{k}$ of $X$. We shall consider then the sets

$$
Z_{k+r}=\left(T-\bar{X}_{k+r}\right) \cup\left(W_{k+r}-V_{n}\right),
$$

where $W_{k+r}$ is the subset of $\bigcup_{l=0}^{\infty} V_{l}$ consisting of all joints of chaplets of orders $<k+r$ and all initial points lying on the boundary of $T$ and on these chaplets. Hence $Z_{k+r}$ is formed by the interior of all discs of the chaplets of orders $<k+r$, all the initial points lying on the boundary of $T$ and on these dises, and all joints between these dises. except those belonging to $V_{n}$.

A good deal of our considerations will be restricted to the common part of $Z_{k \div r}$ with the triangle $T_{v_{k}}$ for some $v_{k} \in N_{k}$, i.e. with the set

$$
Z_{v_{k}, r}=\left(T_{v_{k}}-X_{k+r}\right) \cup\left(T_{v_{k}} \cap W_{k+r}-V_{n}\right) .
$$

Since, in particular, it can be $k=0$, then this case covers also the case $Z_{k+r}$.

Thus, for instance, $Z_{v_{k}, 0}$ is a countable and dense subset of $\operatorname{Fr}\left(T_{v_{k}}\right)$ consisting of some joints of those 3 chaplets, the subsets of which the sides of $T_{v_{k}}$ are, and of the all initial points lying on $\operatorname{Fr}\left(T_{v_{k}}\right)$. The set $Z_{\boldsymbol{v}_{k}, 1}$ consists of $Z_{\boldsymbol{v}_{k}, 0}$ enlarged for the links of 3 chaplets $P_{\boldsymbol{v}_{k}}^{i}(i=1,2,3)$ of order $k$ lying in $T_{v_{k}}$. Adding to $Z_{v_{b}, 1}$ all the links of 12 chaplets $P_{v_{k} j}^{l}$

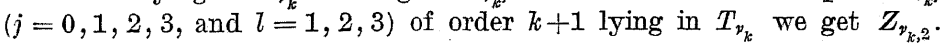
And so on.

In particular, $\overline{Z_{v_{k}, 0}}=\operatorname{Fr}\left(T_{v_{k}}\right), \overline{Z_{v_{k}, 1}}=\operatorname{Fr}\left(T_{v_{k}}\right) \cup \bigcup_{i=1}^{3} P_{v_{k}}^{i}$ and, generally, $\overline{Z_{v_{k}, r}}$ is the union of boundary of $T_{\nu_{k}}$ and all chaplets of $T_{\nu_{k}}$ of orders $<k+r$.

Returning to $Z_{v_{k}, 2}$ notice that since 12 chaplets $P_{\boldsymbol{v}_{k} j}^{l}$ have only their extreme points common with the closure of $Z_{v_{k}, 1}$ then each of their links, different from the first, is a component of $Z_{v_{k}, 2^{2}}$ and, consequently, the only components of $Z_{y_{k^{2}}}$ are:

(i) each point of $Z_{y_{k}, 2}$, which lies on $\operatorname{Fr}\left(T_{v_{k}}\right)$ and is not the initial of any chaplet $P_{\boldsymbol{y}_{k}}^{i}$ or $P_{\boldsymbol{v}_{k} j}^{l}$;

(ii) unions of first link of a chaplet $P_{\boldsymbol{v}^{j}}^{l}$ with some link of a chaplet $P_{\boldsymbol{v}_{k}}^{i}$ (namely with the containing the initial point $v_{\boldsymbol{v}_{k}, 1}^{l}$ of $P_{\boldsymbol{v}_{k} j}^{l}$ );

(iii) all other links of 15 chaplets $P_{v_{k}}^{i}$ and $P_{v_{k} j}^{l}$. 
Similarly, passing from $Z_{v_{k}, 2}$ to $Z_{v_{k}, 3}$ we find that only those components of $Z_{v_{k^{2}}}$, which contains the initials of chaplets of order $k+2$, can be enlarged.

Denoting then by $C_{v_{k}, s}(a)$ a component of a point $a \in Z_{k_{k}, s}$, we have in general

6.1. If $a \in Z_{\psi_{k}, s}$, then

$$
C_{v_{k}, s+1}(a)=C_{v_{k}, s}(a) \cup \bigcup_{v_{v k+\varepsilon}^{j} \in O_{v_{k, e}}(a)} P_{v_{k+s, 1}}^{j} .
$$

This lemma has some important consequences.

Notice first that passing from $C_{p_{k}, r}(a)$ to containing it $C_{p_{k}, r+1}(a)$ we enlarge the first for links of chaplets not contained in $Z_{v_{k}, r}$ but having its initials in $C_{y_{k}, r}(a) \subset Z_{v_{k}, r}$, and therefore $C_{v_{k}, r+1}(a) \cap Z_{v_{k}, r}$ $=C_{v_{k}},(a)$, whence by an obvious induction, we obtain:

6.2. If $a \in Z_{v_{k^{\prime}}, r}$ and $r \leqslant s$, then $C_{v_{k}, s}(a) \cap Z_{v_{k}, r}=C_{v_{k}, r}(a)$.

Secondly, we have

6.3. If $a \in Z_{v_{k^{\prime}},}$, then $C_{v_{k,}, s}(a)$ is a point or a union of some links of chaplets of orders $<k+s$ contained in $T_{v_{k}}$ and each such chaplet has at most one link common with $C_{\boldsymbol{v}_{k}, s}(a)$. More exactly, if $r_{0}$ is the first natural number such that $Z_{v_{k}, r_{0}} \cap C_{v_{k}, s}(a) \neq 0$, then

(a) $Z_{v_{k}, r_{0}} \cap C_{\boldsymbol{v}_{k}, s}(a)$ is a point for $r_{0}=0$ and one, but not first, link of some chaplet of order $k+r_{0}-1$ for $r_{0}>0$;

(b) if $r_{0} \leqslant r \leqslant s$, then $C_{v_{k}, s}(a) \cap\left(P_{v_{k+r}}^{i}-v_{v_{k+r^{1}}}^{i}\right)$ is empty or equal to first link $P_{v_{k+r^{1}}}^{i}$.

Proof. (a) If $r_{0}=0$, then $C_{v_{k}, s}(a) \cap Z_{v_{k}, 0}$ is a point, because $Z_{v_{k}, 0}$ is a countable subset of $\operatorname{Fr}\left(T_{v_{k}}\right)$.

And if $r_{0}>0$, then $C_{v_{k} s}(a)$ meets some chaplet $P_{v_{k+r_{0}-1}}^{i}$ and does not meet $Z_{v_{k}, r_{0}-1}$. Since first link of this chaplet has common initial point $v_{v_{k+r_{0}-1,1}}^{i}$ with $Z_{v_{k}, r_{0}-1}$, then $P_{v_{k+r_{0}-1}}^{i} \cap C_{w_{k}, s}(a)$ contains one of the next links of $P_{v_{k+r_{0}-1}}^{i}$. And since each link of $P_{v_{k+r_{0}-1}}^{i}$ except the first is a component of $Z_{v_{k}, r_{0}}$, and $Z_{y_{k}, r_{0}} \cap C_{\eta_{k}, s}(a)$ is connected by 6.2 , then it consists of this link only.

(b) follows by 6.1 and 6.2 .

6.4. If $a \in Z_{\eta_{k}, 0}$, then $\overline{C_{v_{k}, s}(a)}$ is the union of closures of first links contained in $C_{\eta_{k, s}}(a)$. In particular, if $\overline{V_{v_{k}, s}(a)} \cap\left(P_{v_{k+r}}^{i}-v_{v_{k+r}, 1}^{i}\right) \neq 0$, then $v_{v_{k+p, i}}^{i} \in C_{v_{k, s}}(a)$.

Proof. Since $C_{v_{k}, s}(a)$ consists of finitely many first links (as there is only finitely many chaplets of orders $<k+s)$, then its closure is the union of closures of these links.
6.5. For each $P_{v_{k+r}}^{i} \subset T_{v_{k}}$ there is at most one point $a \in Z_{v_{k}, 0}^{i}$ such that $\overline{C_{v_{k}, r}(a)} \cap\left(P_{v_{k+r}}^{i}-v_{v_{k+r}, \omega}^{i}\right) \neq 0$.

Proof is any easy induction on $r=0,1, \ldots$, because initial point $v_{v_{k+r}, 1}^{i}$ of $P_{v_{k+r}}^{i}$ lies on the boundary of $T_{v_{k}}$ or on the chaplet of order $<k+p$, and the assumed inequality implies, by 6.3 , that $v_{v_{k+r}, 1}^{i} \in C_{v_{k}, r-1}(a)$.

6.6. If $a=v_{v_{k+p}, 1}^{i}$ for some $p=0,1, \ldots, v_{k+p} \in N_{k+p}$ and $i=1,2,3$, then $\overline{C_{v_{k}, r}(a)} \subset(a) \cup \operatorname{Int}\left(T_{\boldsymbol{v}_{k+p}}\right)$ for each $r=0,1, \ldots$

Proof. By 6.1, we have $C_{v_{k}, r}(a)=a$ for $r=0,1, \ldots, p$ and $C_{v_{k}, p+1}(a)$ $=P_{v_{k+p}, 1}^{i}$. Since the chaplet $P_{\boldsymbol{v}_{k+p}}^{i}$, which divorces (by its definition) 2 subtriangles $T_{v_{k+p} 0}$ and $T_{\boldsymbol{v}_{k+p}}$ of $T_{\boldsymbol{v}_{k+p}}$, lies entirely in $\operatorname{Int}\left(T_{\boldsymbol{v}_{k+p}}\right)$ except its extreme points, then, in view of $v_{v_{k+p}, 1}^{i}=a$ and $v_{v_{k+p}, \omega}^{i} \overline{P_{v_{k+p}, 1}^{i}}$, we have 6.6 also for $r=p+1$. Now, the inductive proof follows by (37).

The next lemma is valid for all $k$, but we shall use and therefore formulate it for the case $k=0$ only.

6.7. Let $s=1,2, \ldots, a \in Z_{s}, b \in Z_{s}$ and $a \neq b$. Then

(1) $X-\overline{C_{v_{0}, s}(a)}$ is dense in $X$,

(2) $X-\overline{C_{\nu_{0}, s}(a)}$ is connected,

(3) if $C_{v_{0}, s}(a) \cap C_{v_{0}, s}(b)=0$, then $\overline{{V_{v}, 8}_{s}(a)} \cap \overline{C_{v_{0}, s}(b)} \subset V_{n}$.

Proof. (1) By 6.4, $\overline{C_{v_{s}, s}(a)}$ is a union of closures of finitely many links and therefore of finitely many dises of chaplets of orders $<s$. Since no internal point of any of these discs belongs to $X$, then $X$ can meet $\overline{C_{v_{0}, s}(a)}$ in boundaries of these discs only. And since these boundaries are parts of boundaries of chaplets containing these discs, then $X \cap$ $\cap \overline{C_{v_{0}, s}(a)} \subset \operatorname{Fr}\left(X_{\delta}\right)$, because, by $(25), \operatorname{Fr}\left(X_{\delta}\right)$ is a union of $\operatorname{Fr}\left(X_{0}\right)$ and boundaries of chaplets of order $<s$. Now, $\operatorname{Fr}\left(X_{\delta}\right)$ is, by (27); boundary in $X$ and so is its subset.

(2) Note first that in view of 6.3 the set $\overline{C_{v_{0}, s}(a)}$ does not contain all joints of any chaplet of order $<s$.

Hence and from $(40)$ we infer $\left({ }^{10}\right)$ that $X_{s}-\overline{C_{\gamma_{0}, s}(a)}$ is connected. In view of (15) we have $X_{s}-\overline{C_{v_{0}, s}(a)}=\bigcup_{v_{s} \in N_{s}}\left(T_{v_{s}}-\overline{C_{v_{0}, s}(a)}\right)$. Now replace each sommand on the right hand by $T_{v_{8}} \cap X-\overline{C_{v_{0}, 8}(a)}$, which is connected by (34), because $\overline{C_{\nu_{0}, s}(a)} \cap T_{v_{s}} \subset \operatorname{Fr}\left(T_{\nu_{s}}\right)$ for each $\nu_{s} \in N_{s}$. And since $\operatorname{Fr}\left(T_{v_{s}}\right)-\overline{C_{\nu_{0}, s}(a)} \subset T_{v_{s}} \cap X-\overline{C_{v_{0}, s}(a)}$, because $\operatorname{Fr}\left(T_{v_{s}}\right) \subset X$, then the set $\bigcup_{v_{s} \in N_{s}} T_{v_{s}} \cap X-\overline{C_{v_{0}, s}(a)}=X-\overline{C_{v_{0}, s}(a)}$ is connected.

( $\left.{ }^{10}\right) \overline{C_{v_{0}, 8}(a)}$ might separate $X_{8}$ between, say, $p$ and $q$ without separating the plane, but in such a case there exists continuum $K$ separating plane between $p$ and $q$ and such that $K \cap X_{8} \subset \overline{C_{v_{0}, 8}(a)}$. 
(3) Each of the sets $\overline{C_{y_{0}, s}(a)}$ and $\overline{C_{v_{0}, s}(b)}$ is, by 6.4 , a finite union of dises of chaplets of order $<s$, and then, if these 2 sets meet, there exist 2 discs $D_{1} \subset \overline{C_{v_{0}, s}(a)}$ and $D_{2} \subset \overline{C_{v_{0}, s}(b)}$ such that $D_{1} \cap D_{2} \neq 0$. If these discs belong to the same chaplet, then $D_{1} \cap D_{2}$ is a joint of this chaplet, and if they belong to different chaplets, then one of them has order inferior than the other (because no 2 discs of 2 chaplets of the same order can meet), but since this other chaplet can meet the union of chaplets of inferior orders in its extreme points only and no one of its disc contains its final point, then $D_{1} \cap D_{2}$ is an initial point of the second chaplet. So, in any case,

$$
D_{1} \cap D_{2} \in W_{s} \cap \bigcup_{l=0}^{\infty} V_{l} .
$$

If the point $p=D_{1} \cap D_{2}$ does not belong to $V_{n}$, then it belongs to $Z_{s}$ and therefore $\operatorname{Int}\left(D_{1}\right) \cup \operatorname{Int}\left(D_{2}\right) \cup(p)$ is a connected subset of $Z_{s}$. Since $\operatorname{Int}\left(D_{1}\right) \subset C_{v_{0}, s}(a), \operatorname{Int}\left(D_{2}\right) \subset C_{v_{0}, s}(b)$ and both $C_{v_{0}, s}(a)$ and $C_{v_{0}, s}(b)$ are components of $Z_{s}$, then $\operatorname{Int}\left(D_{1}\right) \cup \operatorname{Int}\left(D_{2}\right) \cup(p)$ is contained in both $C_{v_{0}, s}(a)$ and $C_{v_{0}, s}(b)$, which is a contradiction to hypothesis. to $V_{n}$.

It means that each common point of $\overline{C_{\nu_{0}, s}(a)}$ and $\overline{C_{\gamma_{0}, 8}(b)}$ belongs

$$
\begin{aligned}
& \text { 6.8. If } a \in Z_{k} \text { and } 0<k<s \text {, then } \\
& \qquad C_{v_{0}, s}(a)=C_{v_{0}, k}(a) \cup \bigcup_{v_{k} \in N_{k} x \in C_{v_{0}, k}(a) \cap T_{v_{k}}} C_{v_{k}, s-k}(x) .
\end{aligned}
$$

In fact, by virtue of 6.1, $C_{p_{0}, s}(a)$ is obtained from $C_{p_{0}, s}(a)$ by successive enlargement $C_{v_{0}, k}(a)$ to $C_{v_{0}, k+1}(a)$, next to $C_{v_{0}, k+2}(a)$ and so on until $C_{v_{0}, s}(a)$. And we enlarge $C_{v_{0}, k}(a)$ to $C_{v_{0}, k+1}(a)$ by joining to $C_{v_{0}, k}(a)$ all the components of points $x \in C_{v_{0}, k}(a) \cap T_{v_{k}}$ in $Z_{v_{k}, 1}$, where $v_{k} \in N_{k}$. And enlarging the last components to the components $C_{v_{k}, 2}(x)$ is $Z_{y_{k}, 2}$ we get $C_{v_{0}, k+2}(a)$, and so on until enlarging them to the components $C_{\boldsymbol{v}_{k}, s-k}(x)$ in $Z_{\boldsymbol{p}_{k}, s-k}$ we finally get $C_{v_{0}, s}(a)$.

6.9. If $k \geqslant n$ and $x \in Z_{v_{k^{0}},}$, then for each $r=0,1, \ldots$ there exists a connected set $A_{r}$ such that

(*) $\quad T_{\boldsymbol{v}_{k}} \cap \operatorname{Fr}\left(X_{k+r}\right)-\overline{C_{\boldsymbol{v}_{k^{\prime}}}(x)} \subset A_{r} \subset T_{v_{k}} \cap \operatorname{Fr}\left(X_{k+r+1}\right)-\overline{C_{v_{k}, r+1}(x)}$.

Proof. If $x \in Z_{v_{k}, 0}-\nabla_{0}$, then by 6.1, $C_{v_{k}, r}(x)=x$ for $r=0,1, \ldots$ and therefore putting $A_{r}=T_{\boldsymbol{v}_{\boldsymbol{k}}} \cap \operatorname{Fr}\left(X_{k+r}\right)-(x)$ we have 6.9 by (32).

And if $x=v_{v_{k+s}, 1}^{i}$ for some $s=0,1, \ldots, v_{k+s} \in N_{k+s}$ and $i=1,2,3$, then by $6.1, G_{v_{k}, r}(x)=x$ for $r=0,1, \ldots, s$ and therefore, in view of $(32)$,
the sets

$$
A_{r}=T_{\boldsymbol{v}_{k}} \cap \operatorname{Fr}\left(X_{k+1}\right)-\overline{{V_{k_{k}}, r}(x)}
$$

are connected and satisfy $(*)$ for $r=0,1, \ldots, s$.
The rest of the proof follows by induction. Supposing that there exists a set $A_{r}$ satisfying (*), we shall construct a set $A_{r+1}$ satisfying (*) for $r+1$ instead of $r$. For that purpose consider the set

$$
B_{r \div 1}=T_{v_{k}} \cap \operatorname{Fr}\left(X_{k+r \div 1}\right)-\overline{C_{v_{k}, r+1}(x)} .
$$

By $(*), A_{r} \subset B_{r+1}$.

If $B_{r+1}$ is connected, then put $A_{r+1}=B_{r+1}$.

If $B_{r+1}$ is not connected, then first notice that

(a) if component $H$ of the set $B_{r+1}$ is disjoint with $A_{r}$, then

$H=\operatorname{Fr}\left(P_{\boldsymbol{v}_{k+r}}^{i}\right)-\left(\overline{P_{\boldsymbol{v}_{k+r}, 1}^{i}} \cup v_{\boldsymbol{v}_{k+r}, \omega}^{i}\right) \quad$ for some $\quad v_{k+r} \in N_{k+r}$ and $i=1,2,3$.

Indeed, since $B_{r+1}-A_{r} \subset T_{v_{k}} \cap\left[\operatorname{Fr}\left(X_{k+r+1}\right)-\operatorname{Fr}\left(X_{k+r}\right)\right]$, then, by (25), $B_{r \div 1}-A_{r} \subset \bigcup_{v_{k+r} \in N_{k+r}} \bigcup_{i=1}^{3} P_{\boldsymbol{v}_{k+r}}^{i}$ and therefore each component $H$ of $B_{r+1}$, which is disjoint with $A_{r}$, is a subset of some chaplet $P_{v_{k+r}}^{i}$.

Consider then an arbitrary chaplet $P_{v_{k+r}}^{i}$ of $T_{v_{k}}$.

If its initial point $v_{v_{k+r}, 1}^{i}$ does not belong to $C_{v_{k}, r}(x)$, then, by 6.4, $\overline{C_{p_{k}, r+1}(x)} \cap\left(P_{\boldsymbol{v}_{k+r}}^{i}-v_{\boldsymbol{v}_{k+r}, w}^{i}\right)=0$. And since the set $\operatorname{Fr}\left(P_{\boldsymbol{v}_{k+r}}^{i}\right)-v_{\boldsymbol{v}_{k+r}, \omega}^{i}$ is connected and contains $v_{\boldsymbol{v}_{k+r}, 1}^{i}$, which at the same belongs, by induction hypothesis, to $A_{r}$, then $A_{r} \cup\left[\operatorname{Fr}\left(P_{v_{k+r}}^{i}\right)-v_{v_{k+r}, \omega}^{i}\right]$ is connected subset of $B_{r+1}$.

And if $v_{v_{k+r}, 1}^{i} \in C_{v_{k}, r}(x)$, then, by 6.3 and 6.4 ,

$$
\overline{C_{v_{k}, r+1}(x)} \cap\left(P_{\boldsymbol{v}_{k+r}}^{i}-v_{v_{k+r}, \omega}^{i}\right)=\overline{P_{v_{k+r}, 1}^{i}}
$$

Now all depends on whether the final point $v_{v_{k+r}, \omega}^{i}$ belongs or not to $C_{v_{k}, r}(x)$. If it does not, then by induction hypothesis it belongs to $A_{r}$ and since the set $\operatorname{Fr}\left(P_{\boldsymbol{v}_{k+r}}^{i}\right)-\overline{P_{\boldsymbol{v}_{k+r}, 1}^{i}}$ is connected and contains $v_{\boldsymbol{v}_{k+r}, \omega}^{i}$, then $A_{r} \cup \operatorname{Fr}\left(P_{v_{k+r}}^{i}\right)-\overline{P_{v_{k+r}, 1}^{i}}$ is connected subset of $B_{r+1}$. But if it does, then $\operatorname{Fr}\left(P_{v_{k+r}}^{i}\right)-\left(P_{v_{k+r}, 1}^{i} \cup v_{v_{k+r}, \omega}^{i}\right)$ is connected and disjoint with $C_{v_{k}, r+1}(x)$ subset of $B_{r+1}$.

Hence (a) is proved and for each such component $H$ we shall now construct in the set $B_{r+2}$ and arc $L_{H}$ joining $H$ with $A_{r}$.

For that purpose consider triangle $T_{v_{k+r}}$ (see Fig. 12) and first notice that neither side of $T_{v_{k+r}}$ containing initial point $v_{v_{k+r}, 1}^{i}$ nor side containing final point $v_{\boldsymbol{v}_{k+r} \omega}^{i}$ of $P_{\boldsymbol{v}_{k+r}}^{i}$ is a subset of a chaplet divorcing triangle $T_{v_{k+r}}$ from some other triangle of $X_{k+r}$. For if so, then by (20), (42) and hypothesis $k \geqslant 2$ the extreme point of $P_{\boldsymbol{v}_{k+r}}^{i}$ lying on such a side could not belong to the first link of corresponding chaplet and Fundamenta Mathematicae, T. LVIr 
therefore, by 6.3 and 6.4 , it could not belong to $\overline{C_{\nu_{k}, r}(x)}$, but both extreme points of $P_{v_{k+r}}^{i}$ do.

Hence the third side of $T_{v_{k+r}}$ must be a subset of a chaplet divorcing $T_{v_{k+r}}$ from some other triangle of $X_{k+r}$ and therefore the initial point $v_{v_{k+r}, 1}^{i}$ of the chaplet $P_{v_{k+r}}^{i}$ joining this side with initial of $P_{v_{k+r}}^{i}$ does not belong to $C_{v_{k}, r}(x)$, whence, in view of induction hypothesis,

(b) $v_{v_{k+r^{1}}}^{i} \in A_{r}$.

Let now $P_{\boldsymbol{v}_{k+r} 0}^{l}$ be a chaplet of triangle $T_{v_{k+r^{0}}}$, the initial point of which lies in $P_{v_{k+r}}^{i}$. From (b) and 6.4 we infer that $\left(P_{v_{k+r}}^{j}-v_{v_{k+r}, \omega}^{j}\right) \cap$

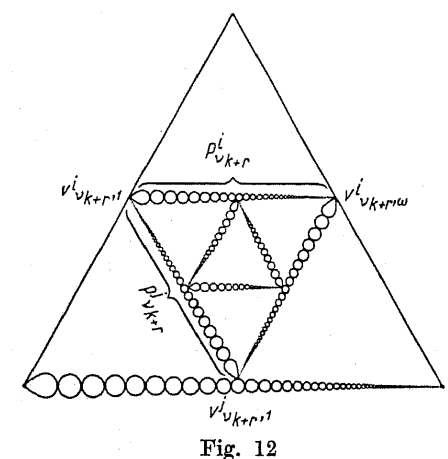

$\cap C_{\boldsymbol{y}, r+2}(x)=0$ and $P_{\boldsymbol{v}_{k+r} 0}^{l} \cap C_{\boldsymbol{v}_{k}, r+2}(x)=0$. Then an arc $L_{H} \subset P_{\boldsymbol{v}_{k+r}}^{j} \cup P_{v_{k+r^{0}}}^{i}$ of ends $v_{v_{k+r}, 1}^{j}$ and $v_{v_{k+r}, 1}^{l}$ is a subset of $B_{r+2}$ and joins $v_{v_{k+r}, 1}^{j} \in A_{r}$ with $v_{v_{k+r}}^{l}, i \in H$.

Hence $A_{r+1}=B_{r+1} \cup \cup L_{H}$, where $H$ runs over all components of $B_{r+1}$ disjoint with $A_{r}$, satisfies (*).

6.10. If $k \geqslant n, r=1,2, \ldots$ and $T_{v_{k+r+1}} \subset \operatorname{Int}\left(T_{v_{k}}\right)$, then there exists at most one point $x \in Z_{v_{k^{0}}}$ such that

$$
T_{\boldsymbol{v}_{k+r+1}} \cap \overline{\widehat{V}_{\boldsymbol{k}, r+1}(x)} \neq 0 .
$$

Proof. Write $v_{k+r+1}$ more explicitly as $v_{k+r-1} i j \quad(i, j=0,1,2,3)$ and consider all possible cases.

1. If $j \neq 0$, then, according to our notations, triangle $T_{v_{k+r-1} i j}$ is divorced from the central triangle $T_{v_{k+r-1} i 0}$ by the chaplet $P_{v_{k+r-1}}^{j}$. Let us denote briefly the side of $T_{v_{k+r-1} i j}$ common with $P_{v_{k+r-1} i}^{j}$ by $A$, the initial and final points of $P_{v_{k+r-1}}^{j}$ by $a$ and $c$, respectively. Hence $a$ and $c$ are the ends of $A$, and $A$ is oriented from $a$ to $c$. Next denote the third vertex of triangle $T_{v_{k+r-1} i j}$ by $b$, its side of ends $a$ and $b$ by $B$, and side of ends $b$ and $a$ by $C$. Denote also shortly by $P_{A}, P_{B}$ and $P_{C}$ chaplets, the subsets of which sides $A, B$ and $O$ respectively are (cf. (35)). So, for instance, $P_{A}=P_{v_{b+1}}^{j}$, and $a$ is neither initial nor final point of $P_{B}$, because $B$ is a "half" of side of triangle $T_{v_{k+r-1} i}$ and this side lies entirely in $P_{B}$ (cf. (35)).

According to (36), $b$ is initial or final point of one of the chaplets $P_{B}$ and $P_{C}$. By virtue of consistent orientation, $b$ is initial of $P_{B}$ or final of $P_{C}$, and these cases do not exclude one another. (Indeed, if $T_{v_{k+r-1}{ }^{i}}$ is itself central, i.e. $i=0$, then $b$, being one of its vertices, is simultaneously initial of $P_{B}$ and final of $P_{C}$.)

(a) If $b$ is final of $P_{C}$ (irrespective of whether it is initial of $P_{B}$ or not) (see Fig. 13), then by (20), (42) and hypothesis $k \geqslant n$, the closure

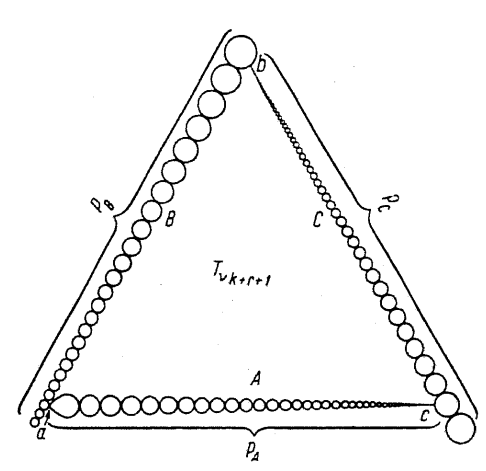

Fig. 13

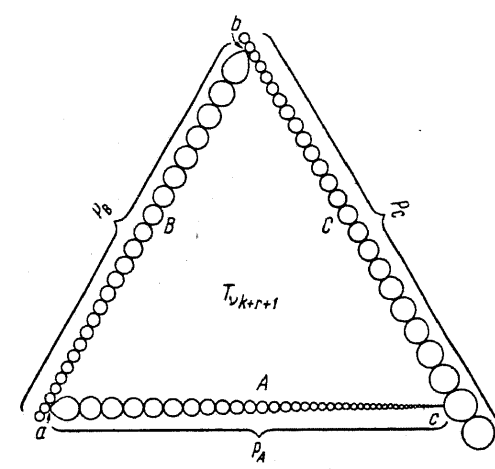

Fig. 14 of first link of $P_{C}$ does not contain $\theta$ and therefore does not meet $C$, which means, in view of 6.4 that

$$
[C-\{b\}] \cap \overline{C_{v_{k}, r+1}(x)}=0 \text { for all } x \in \operatorname{Fr}\left(T_{v_{k}}\right) .
$$

Now, if $A \cap \overline{{v_{k}, r+1}_{1}(x)} \neq 0$ for some $x \in \operatorname{Fr}\left(T_{v_{k}}\right)$, then $a \in \overline{C_{v_{k}}, r(x)}$ and since $a$ is not a final point of $P_{B}$, then $a$ belongs to the closure of first link of $P_{B}$. Hence, by 6.5, $B \cap \overline{C_{p_{b}}, r+1}(y) \neq 0$ iff $y=x$.

And if $A \cap \overline{C_{v_{k}}, r+1}(x)=0$ for each $x \in \operatorname{Fr}\left(T_{v_{k}}\right)$, then only side $B$ remains "free" and we simply apply 6.5 to $P_{B}$.

(b) If $b$ is not final point of $P_{C}$, then $b$ must be initial point of $P_{B}$ (see Fig. 14). By (20), (42) and hypothesis $k \geqslant n$, the elosure of first link of $P_{B}$ does not contain $a$, and then, by 6.4

$$
[A-\{c\}] \cap \overline{C_{\boldsymbol{v}_{k}, r+1}(x)}=0 \text { for each } x \in \operatorname{Fr}\left(T_{\boldsymbol{v}_{k}}\right) .
$$


Now, if $B \cap \overline{C_{v_{k}, r+1}(x)} \neq 0$ for some $x \in \operatorname{Fr}\left(T_{v_{k}}\right)$, then, by 6.4, $b \in \overline{C_{p_{k}, r}(x)}$. But since in view of consistent orientation $P_{C}$ goes in direction from $e$ through $b$, then, by $6.4 b$ is contained together with the side $C$ in the closure of first link of $P_{C}$ and therefore in $\overline{V_{v_{0}},(x)}$. Hence and from 6.5 we infer that $C \cap \overline{C_{v_{k}, r+1}(y)} \neq 0$ iff $y=x$.

And if $B \cap \overline{\sigma_{v_{k}}, r+1}(x)=0$ for each $x \in \operatorname{Fr}\left(T_{v_{k}}\right)$, then only side $C$ remains "free" and we simply apply 6.5 to $P_{C}$.

2. If $j=0$, then also 2 cases are possible.

(a) If $i \neq 0$, then, according to our notations, $T_{v_{k+r-1} i}$ is divorced from the central triangle $T_{v_{k+r-1}{ }^{0}}$ by the chaplet $P_{v_{k+r-1}{ }^{0}}^{i}$ (see Fig. 15).

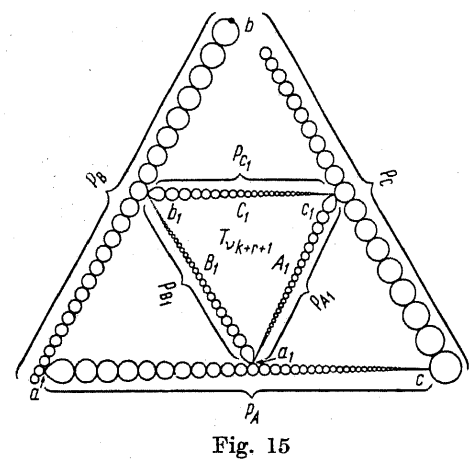

Let us denote the sides, vertices and corresponding chaplets for triangle $T_{v_{k+r-1} i}$ in the same way as we did for triangle $T_{v_{k+r-1} i^{j}}$ in 1 . Denote also the vertices of triangle $T_{v_{k+r-1}}$ lying on $P_{A}, P_{B}, P_{C}$ by $a_{1}, b_{1}, c_{1}$ respectively, the sides of ends $\left(a_{1}, b_{1}\right),\left(b_{1}, c_{1}\right)$ and $\left(c_{1}, a_{1}\right)$ by $B_{1}, C_{1}$ and $A_{1}$ respectively, and corresponding chaplets by $P_{B_{1}}, P_{C_{1}}, P_{A_{1}}$.

Applying (20) and 6.4 to $P_{A}$ we conclude that $a_{1}$ does not belong to $\overline{C_{v_{k}, r}(x)}$ for any $x \in \operatorname{Fr}\left(T_{v_{k}}\right)$ and, consequently, that

$$
\left[B_{1}-\left\{b_{1}\right\}\right] \cap \overline{v_{v_{k}, r+1}(x)}=0 \text { for each } x \in \operatorname{Fr}\left(T_{v_{k}}\right) .
$$

Now, if $b$ is a final point of $P_{C}$, then, similarly to $1(a), c_{1}$ does not belong to $\overline{C_{v_{k}}, r(x)}$ for any $x \in \operatorname{Fr}\left(T_{v_{k}}\right)$, and therefore

$$
\left[A_{1}-\left\{a_{1}\right\}\right] \cap \overline{C_{v_{k}}, r+1}(x)=0 \text { for each } x \in \operatorname{Fr}\left(T_{v_{k}}\right) .
$$

For one remaining "free" side $C_{1}$ we apply 6.5.

And if $b$ is not a final point of $P_{C}$, then it must be initial point of $P_{B}$. Therefore, if

$$
\left[C_{1}-\left\{c_{1}\right\}\right] \cap \overline{C_{v_{k}, r+1}(x)} \neq 0 \text { for some } \quad x \in \operatorname{Fr}\left(T_{v_{k}}\right),
$$

then $b_{1}$ belongs to the closure of first link of $P_{B}$, this link is contained in $\overline{C_{p_{b}, r+1}(x)}$ and, consequently, $b$ belongs to the closure of first link of $P_{C}$. But since $P_{C}$ goes in direction from $c$ through $b$ (in view of consistent orientation), then also $c_{1} \in \overline{C_{v_{k}}, r+1}(x)$. In view of 6.4 it means that

$$
\left[A_{1}-\left\{a_{1}\right\}\right] \cap \overline{V_{v_{k}, r+1}(y)} \neq 0 \text { if and only if } y=x .
$$

(b) The case $i=0$ is simple, because, preserving notations of the case (a) above, each vertex of $T_{v_{k+r-1} i}$ is now simultaneously initial and final of 2 of chaplets $P_{A}, P_{B}, P_{C}$, which implies, by (20), (42) and hypothesis $k \geqslant n$, that $a_{1}, b_{1}, c_{1}$ does not belong to the closures of first links of chaplets $P_{A}, P_{B}, P_{C}$ respectively, whence, in view of 6.4 ,

$$
T_{\boldsymbol{v}_{k+r-1} i j} \cap \overline{C_{\boldsymbol{v}, r+1}(x)}=0 \quad \text { for each } \quad x \in \operatorname{Fr}\left(T_{\boldsymbol{v}_{k}}\right) .
$$

All cases being considered, 6.10 is proved.

Let us pass now to another eategory of sets.

If $x \in Z_{v_{k}, 0}$, then $C_{v_{k}, r}(x)$ is, by the definition, a component of $x$ in the set $Z_{v_{k}, r}$. Tending with $r$ to infinity let us denote by $C_{v_{k}}(x)$ the union

$$
C_{v_{k}}(x)=\bigcup_{r=0}^{\infty} C_{v_{k}, r}(x) \text {. }
$$

One can prove that the set $C_{v_{b}}(x)$ is a component of $x$ in $T_{v_{b}} \cap Z$. For us, however, more important are other properties of it.

Since we get $C_{y_{k}}(x)$ by successive enlarging of a point $x$ to $C_{v_{k}, 1}(x)$, next to $C_{v_{k}, 2}(x)$, and so on, then obviously

6.11. If $x \in Z_{v_{k}, 1}$, then for each $r=0,1, \ldots$

$$
C_{y_{k}}(x) \cap Z_{v_{k}, r}=C_{v_{k}, r}(x) .
$$

Similarly, 6.3 and 6.6 are valid for $C_{v_{k}}(x)$ instead of $C_{v_{k}, s}(x)$, i.e.

6.12. If $x \in Z_{v_{k}, 0}$, then $C_{v_{k}}(x)$ is a union of some links of chaplets contained in $T_{v_{k}}$ and each such chaplet has at most its first link common with $C_{v_{k}}(x)$. More exactly,

$$
C_{v_{k}}(x) \cap\left(P_{\boldsymbol{v}_{i s}}-v_{v_{s}, \omega}^{i}\right) \text { is empty or equal to } P_{\boldsymbol{v}_{s}, 1}^{i} \text {. }
$$

6.13. If $x=v_{v_{k+p}, 1}^{i}$ for some $p=0,1, \ldots, v_{k+p} \in N_{k+p}$ and $i=1,2,3$, then $C_{v_{k}}(x) \subset(x) \cup \operatorname{Int}\left(T_{\eta_{k+p}}\right)$.

6.14. If $k \geqslant n$ and $x \in Z_{y_{k}, 0}$, then for each $r=0,1, \ldots$

$$
T_{v_{k}} \cap \operatorname{Fr}\left(X_{k+r}\right)-\overline{C_{v_{k}, r}(x)}=T_{v_{k}} \cap \operatorname{Fr}\left(X_{k+r}\right)-\overline{C_{v_{k}}(x)} .
$$

Proof. Since $C_{v_{k}, r}(x) \subset C_{v_{k}}(x)$ by (43), then it suffices to prove the inclusion

$$
T_{v_{k}} \cap \operatorname{Fr}\left(X_{k+r}\right)-\overline{C_{v_{k}}, r(x)} \subset T_{v_{k}} \cap \operatorname{Fr}\left(X_{k+r}\right)-\overline{C_{v_{k}}(x)} .
$$


For that purpose let us take a point $p \in T_{v_{k}} \cap \operatorname{Fr}\left(X_{k+r}\right)-\overline{C_{v_{k}, r}(x)}$. Three cases are possible:

(i) $p$ is initial point $v_{v_{k+8}, 1}^{i}$ of some chaplet $P_{v_{k+8}}^{i}$, where $s<r$ (see Fig. 16). It means that $p$ is a vertex of three triangles $T_{v_{k+8} i}, T_{v_{k+8} 0}$ and $T_{v_{k+8}^{j}}$ and that these three triangles together with the first dise $Q_{v_{k+s}, 1}^{i}$ of chaplet $P_{v_{k+s}}^{i}$, with some disc $D$ of chaplet of order $<k+s$ containing $p$, and with the union $\bigcup_{l=p_{k+1}}^{\infty} Q_{v_{k+8}, l}^{i}$ of all discs, beginning from the second link, of chaplet $P_{\boldsymbol{v}_{k+s}}^{i=p_{k+1}}$, form a neighbourhood of $p$.

If $p \in \overline{C_{v_{k}}(x)}$, then $p$ is a cluster point of interiors of dises contained in $C_{v_{k}}(x)$, because the last form the dense subset of $C_{v_{k}}(x)$. But $\operatorname{Int}(D) \cap$

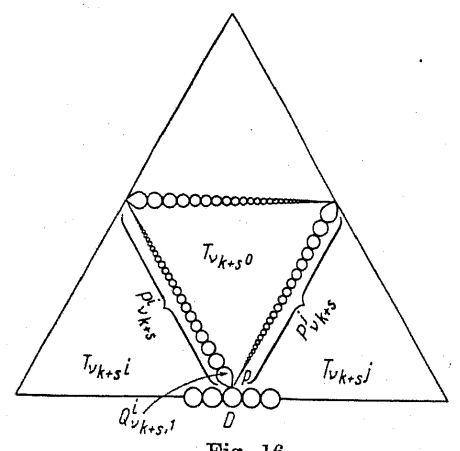

$\cap C_{v_{k}, r}(x)=0$ by hypothesis and therefore, by $6.11, \operatorname{Int}(D) \cap C_{v_{k}}(x)=0$, whence, consequently, also $\operatorname{Int}\left(Q_{v_{k+v_{r}}}^{i}\right) \cap C_{v_{k}}(x)=0$. And since $C_{v_{k}}(x)$ contains, by 6.12, only some first links of chaplets contained in $T_{v_{k}}$, then also $C_{\boldsymbol{v}_{k}}(x) \cap \bigcup_{l=p_{n+1}}^{\infty} \operatorname{Int}\left(Q_{v_{k+8}}^{j}\right)=0$. Hence if $p \in \overline{C_{v_{k}}(x)}$, then $p$ is a cluster point of interiors of dises contained in $C_{v_{k}}(x)$ and lying in one of three triangles $T_{\boldsymbol{v}_{k+q^{a}}}$, where $\alpha=0, i$ or $j$. Take this triangle and consider a triangle $T_{v_{k+q}}$ containing $p$, contained in $T_{v_{k+8} \alpha}$, and such that its sides common with $T_{\boldsymbol{v}_{k+q^{a}}}$ were disjoint with $\overline{C_{v_{k}, r}(x)}$. They are then disjoint with $C_{\boldsymbol{v}_{k}, r}(x)$ and, in view of 6.11 , also with $C_{v_{k}}(x)$. Hence the end points of the third side of $T_{\boldsymbol{v}_{k+q}}$ do not belong to $C_{v_{k}}(x)$ and, consequently, the third side is also disjoint with $C_{v_{k}}(x)$. It means that $\operatorname{Fr}\left(T_{v_{k+q}}\right) \cap C_{v_{k}}(x)=0$ and since $C_{v_{k}}(x)$ is connected, then also $\operatorname{Int}\left(T_{v_{k+q}}\right) \cap \overline{C_{v_{k}}(x)}=0$.

Hence $p$ does not belong to $\overline{C_{v_{k}}(x)}$. (ii) $p$ is a joint $v_{v_{k+\beta}, j}^{i}$ of some chaplet $P_{v_{k+1}}^{i}$, where $s<r$ (see Fig. 17). It means that $p$ belongs to common part of triangles $T_{v_{k+8} 0}$ and $T_{v_{k+8} i}$ (which chaplet $P_{v_{k+s}}^{i}$ divorces by its definition) and that these 2 triangles together with 2 dises $Q_{v_{k+s}, j}^{i}$ and $Q_{v_{k+s}, j+1}^{i}$ form its neighbourhood.

From 6.11 and 6.12 and hypothesis that $p \notin \overline{O_{v_{k}, r}(x)}$ we infer, as in case (i), that $\left[\operatorname{Int}\left(Q_{v_{k+8}, j}^{i}\right) \cup \operatorname{Int}\left(Q_{v_{k+8}, j+1}^{i}\right)\right] \cap C_{v_{k}}(x)=0$. Then, if $p \epsilon \overline{C_{v_{k}}(x)}$, then $p$ is a cluster point of interiors of discs contained in $C_{v_{k}}(x)$ and lying in one of 2 triangles $T_{v_{k+8} a}(\alpha=0$ or $i)$. Take this triangle and, applying (38), consider triangle $T_{v_{k+\alpha}}$ containing $p$, contained in $T_{v_{k+8}}$, and such that both ends of its side containing $p$ lie in $Q_{v_{k+8, j}, j}^{i} \cup Q_{v_{k+8}, j+1}^{i}$ and satisfy (38). Arguing as in the case (i) we find that $\operatorname{Int}\left(T_{v_{k+q}}\right) \cap$ $\cap \overline{C_{v_{k}}(x)}=0$.

It means that also in this case $p$ does not belong to $\overline{C_{v_{k}}(x)}$.

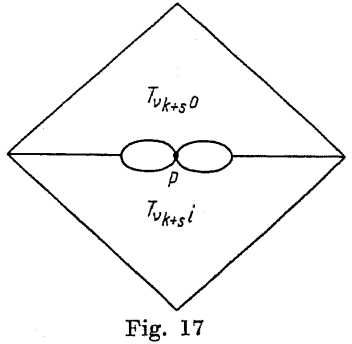

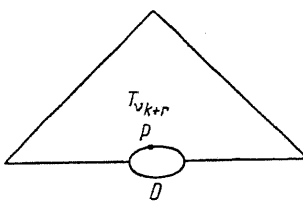

Fig. 18 (iii) if $p$ is neither initial point nor joint of any chaplet $P_{v_{k+\delta}}^{i}$, where $s<r$ (see Fig. 18), then there exists one only dise $D$ and one only triangle $T_{v_{k+r}}$ containing $p$.

Apply argumentation of case (ii) above.

6.15. If $k \geqslant n, r=0,1, \ldots$ and $T_{v_{k+r+1}} \subset \operatorname{Int}\left(T_{v_{k}}\right)$, then there exists at most one point $x \in Z_{v_{k}, 0}$ such that

$$
T_{v_{k+r+1}} \cap \overline{{v_{k}}_{k}(x)} \neq 0 .
$$

In particular, if $x_{1} \in Z_{\psi_{k}, 0}, x_{2} \in Z_{\psi_{k}, 0}$ and $x_{1} \neq x_{2}$, then

$$
\operatorname{Int}\left(T_{v_{k}}\right) \cap \overline{C_{v_{k}}\left(x_{1}\right)} \cap \overline{C_{v_{k}}\left(x_{2}\right)}=0 .
$$

Indeed, if $T_{v_{k+r+1}} \cap \overline{C_{v_{k}}, r+1}(x)=0$ for some $x \in Z_{v_{k}, 0}$, then obviously $\operatorname{Fr}\left(T_{y_{k+r+1}}\right) \cap C_{v_{k}, r+1}(x)=0$ and therefore, by 6.14, $\operatorname{Fr}\left(T_{v_{k+r+1}}\right) \cap \overline{C_{v_{k}}(x)}=\mathbf{0}$. And since $\overline{C_{y_{k}}(x)}$ is connected and $x \in C_{v_{k}}(x)-T_{v_{k+r+1}}$, then finally $T_{v_{k+r+1}}$ ? $\cap C_{v_{k}}(x)=0$. Now apply 6.10. 
6.16. If $k \geqslant n$ and $x \in Z_{v_{k}, 0}$, then $X \cap \overline{\sigma_{v_{k}}(x)}$ is a boundary subset of $X \cap T_{v_{k}}$.

For if $p \in X \cap T_{\nu_{k}}$ and $U$ is a neighbourhood of $p$, then by (15), (17) and (18) there exists $r$ such that $p \in T_{v_{k+r}} \subset U$. At least one side, denote it by $A$, of $T_{v_{k+r}}$ is oriented. Since $\overline{C_{v_{k}, r}(x)}$ contains at most first link of the chaplet, the subset of which $A$ is, then $A-\overline{C_{\nu_{k}, r}(x)} \neq 0$. Hence and from 6.14 we infer that also $A-\overline{V_{v_{k}}(x)} \neq 0$.

6.17. If $A \subset Z_{v_{k}, 0}$, then $\operatorname{Int}\left(T_{v_{k}}\right) \cap \overline{\bigcup_{x \in A} C_{v_{k}}(x)}=\operatorname{Int}\left(T_{v_{k}}\right) \cap \bigcup_{x \in A} \overline{C_{p_{k}}(x)}$.

Indeed, if $x \in Z_{v_{k}, 0}-V_{0}$, then by 6.1 and (43) $C_{\nu_{k}}(x)=x$. And if $x=v_{v_{k+p}, 1}^{i}$ then, by 6.13 ,

(a)

$$
C_{v_{k}}(x) \subset(x) \cup \operatorname{Int}\left(T_{v_{k+p}}\right) .
$$

Since there is only finitely many chaplets of order $\leqslant k+p$, the initials of which lie in $A \cap V_{0}$ (because there is altogether only finitely many chaplets of order $\leqslant k+p$ ), then for each $\varepsilon>0$ there is, in view of (a), (18) and (19), only finitely many sets $O_{v_{k}}(x)$ such that $x \in A$ and $\delta\left[C_{y_{k}}(x)\right] \geqslant \varepsilon$. Each point $p \in \operatorname{Int}\left(T_{v_{k}}\right)$ has then a neighbourhood $U$ meeting only finitely many sets $C_{v_{k}}(x)$ (where $x \in A$ ) and therefore, if $p \in \operatorname{Int}\left(T_{v_{k}}\right) \cap$ $\cap \overline{\bigcup_{x \in A} C_{y_{k}}(x)}$, then $p \in \operatorname{Int}\left(T_{y_{k}}\right) \cap \bigcup_{x \in A} \overline{C_{v_{k}}(x)}$.

The inverse implication is obvious.

As a simple consequence of 6.15 and 6.17 we have

6.18. If $k \geqslant n, A_{1} \cup A_{2} \subset Z_{v_{k}, 0}$ and $A_{1} \cap A_{1}=0$, then

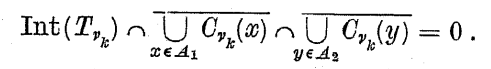

6.19. If $k \geqslant n$ and $x \in Z_{\psi_{k}, 0}$, then $T_{\nu_{k}} \cap X-\overline{C_{\nu_{k}}(x)}$ is a connected and dense subset of $T_{v_{k}} \cap X$.

By virtue of 6.9 the union $\bigcup_{r=0}^{\infty} A_{r}$ of sets satisfying (*) is connected, because obviously $A_{r} \subset A_{r+1}$. And in view of $6.14, \bigcup_{r=0}^{\infty} A_{r}=\bigcup_{r=0}^{\infty} T_{\nu_{k}}$. $\cap \operatorname{Fr}\left(X_{k+r}\right)-\overline{C_{y_{k}}(x)}$. Apply (27).

\$7. Proof of the decomposition (2) of $X$. Now let us return to the set $Z$. The aim which we follow is to represent $Z$ as a union

$$
Z=\bigcup_{k=1}^{\infty} D_{k}
$$

of sets $D_{k}$ having the properties

(45) if $l, m=1,2, \ldots$ and $l \neq m$, then $\overline{D_{k}} \cap \overline{D_{l}} \subset V_{n}$,

(46) for any $l=1,2, \ldots$ and any $R \subset \bar{D}_{l}$ the set $X-R$ is connected.
We shall do it in the following way. Take a point $a \in Z$. Then $a \in Z_{k}$ for some $k=0,1, \ldots$ Now take a component $C_{v_{0}, k}(a)$ of $a$ in $Z_{k}$ and join to it all the sets $C_{v_{k}}(x)$, where $x \in T_{v_{\boldsymbol{k}}} \cap C_{\boldsymbol{v}_{0}, k}(a)$. So we get a connected set

$$
\Delta_{k}(a)=C_{v_{0}, k}(a) \cup \bigcup_{v_{k} \in N_{k}} \bigcup_{x \in T_{v_{k}} \cap C_{v_{0}, k}(a)} C_{v_{k}}(x) .
$$

We shall show that these sets satisfy (44)-(46).

For that purpose notice first that in view of 6.8 the definition of $\Delta_{k}$ (a) depends neither on $k$ nor on $y$ in the sense that if $b \epsilon \Delta_{k}(a) \cap Z_{l}$, then $\Delta_{k}(a)=\Delta_{l}(b)$, and therefore we may, cancelling out some terms, order all the sets $\left\{\Delta_{k}(a)\right\}_{k=0,1, \ldots, a \in Z_{k}}$ in a countable sequence of disjoint sets $\left\{D_{k}\right\}_{k=1,2, \ldots}$. So we have $(44)$.

Now we show (45). Take two different sets $D_{l}$ and $D_{m}$, and, choosing $k$ such that $Z_{k}$ meets both $D_{l}$ and $D_{m}$, take points $a \in Z_{k} \cap D_{l}$ and $b \in Z_{k} \cap D_{m}$. Thus we have

$$
\begin{aligned}
& D_{l}=C_{v_{0}, k}(a) \frown \bigcup_{v_{k} \in N_{k}} \bigcup_{x \in T_{y_{k}} \cap C_{v_{0}, k}(a)} C_{y_{k}}(x), \\
& D_{m}=C_{v_{0}, k}(b) \cup \bigcup_{v_{k} \in N_{k}} \bigcup_{y \in T_{v_{k}} \cap C_{v_{0}, k k}(b)} C_{v_{k}}(y),
\end{aligned}
$$

whence in view of 6.14

$$
\begin{aligned}
& D_{l}=\overline{C_{v_{0}, k}(a)} \cup \bigcup_{v_{k} \in N_{k}} \operatorname{Int}\left(T_{v_{k}}\right) \cap \overline{\underbrace{}_{x \in T_{v_{k}} \cap C_{v_{0}, k}(a)} C_{v_{k}}(\bar{x})}, \\
& \overline{D_{m}}=\overline{C_{v_{0}, k}(b)} \cup \bigcup_{v_{k} \in N_{k}} \operatorname{Int}\left(T_{v_{k}}\right) \cap \overline{\bigcup_{y \in T_{v_{k}} \cap C_{v_{0}, k}(b)} C_{v_{k}}(y)} .
\end{aligned}
$$

Hence by virtue of 6.18 and 6.7 (3),

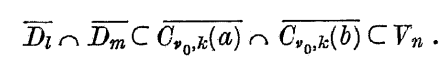

Before proceeding to prove (46) we shall show three preliminary lemmas.

7.1. If $D$ is a dise of some chaplet and $\overline{D_{l}}$ meets boundary of $D$ in more than one point, then $D \subset \overline{D_{l}}$.

For if $D$ is not contained in $\overline{D_{l}}$, then it is contained in some other $\overline{D_{m}}$ and since, by just proved (45), $\overline{D_{l}} \cap \overline{D_{m}} \subset V_{n}$, then $D \cap \overline{D_{l}}$ is either empty or consists of some points of $\nabla_{n}$ belonging to $D$. But each dise of any chaplet contains obviously at most one point of $\nabla_{n}$. (In fact, each disc $Q_{v_{k}, j}^{i}$ contains one only joint $v_{v_{k}, j+1}^{i}$ if $j=1$ or two joints $v_{v_{k}, j}^{i}$ and $v_{v_{k}, j+1}^{i}$ if $j>1$, and has no other common points with $\bigcup_{l=1}^{\infty} V_{l}$ ).

7.2. For each $l=1,2, \ldots: \overline{D_{l}} \cap \operatorname{Fr}(T)$ is empty or consists of one point onty. 
For let $x \in \overline{D_{l}} \cap \operatorname{Fr}(T)$. In view of (47) we have then $D_{l}=C_{\nu_{0}}(x)$ and from 6.13 we infer that $\overline{\sigma_{v_{0}, n}(x)} \cap \operatorname{Fr}(T)=x$, because, by virtue of (22) and $(26), \operatorname{Fr}(T) \subset \bigcup_{v_{k} \in N_{k}} \operatorname{Fr}\left(T_{v_{k}}\right)$. Now apply 6.14 .

7.3. For each $7=1,2, \ldots$ if $X-\overline{D_{l}}$ is not connected between $p$ and $q$, then $\overline{D_{l}}$ contains simple closed curve $S$ separating plane between $p$ and $q$.

For supposing that $X-\overline{D_{l}}=M \cup N$, where $M \cap \bar{N} \cup \bar{M} \cap N=0$, $p \in M, q \in N$, take a continuum $Q$ disjoint with $M \cup N$ and separating the plane between $p$ and $q$ (see [1], p. 233). Since $X$ is connected, then $Q$ must meet $\bar{D}_{l}$ and therefore $Q \cup \overline{D_{l}}$ is continuum. And since $Q$ separates the plane between $p$ and $q$, then continuum $Q \cup \bar{D}_{l}$ does it too.

We shall show that

(a) $\bar{D}_{l}$ itself separates the plane between $p$ and $q$.

Indeed, since $X$ is continuum, $Q$ may have common points with those regions of $E^{2}-X$ only, the boundaries of which have common points with $\overline{D_{l}}$. Take such a region $H$ and suppose first that $\overline{D_{l}} \cap \operatorname{Fr}(H)$ is one point $d$ only. In this case $\overline{D_{l}} \cup Q$ is a union of 2 continua $\bar{D}\urcorner \cup Q-H$ and $d \cup Q \cap H$ joined by the point $d$. Since point $d$ does not separate $X$ (cf. (29)) and $(d \cup Q \cap H) \cap X=d$, then continuum $d \cup Q \cap H$ does not separate $X$ and therefore does not separate plane between $p$ and $q$.

Hence by the First Theorem of Janiszewski (see [4], p. 355) continuum $\overline{D_{l}} \cap Q-H$ separates plane between $p$ and $q$ and so we may remove from $\overline{D_{l}} \cup Q$ the part of $Q$ lying in $H$.

Suppose now that $\operatorname{Fr}(H) \cap \overline{D_{l}}$ contains 2 or more points. In view of $7.2 H$ cannot be unbounded region and therefore $H$ is an interior of a dise of some chaplet. But, in this case, we have $\bar{H} \subset \overline{D_{l}}$ by virtue of 7.1 .

Hence we have proved (a).

Moreover,

(b) $\bar{D}_{l}$ is locally connected continuum.

Indeed, in view of its definition (cf. (47)) we have

$$
\overline{D_{l}}=\overline{C_{v_{0}, k}(a)} \cup \bigcup_{v_{k} \in T_{v_{k}}}\left[T_{v_{k}} \cap \overline{\left.\bigcup_{x \in T_{v_{k}} \cap C_{v_{0}, k}(a)} C_{v_{k}}(\bar{x})\right]}\right.
$$

for some $a \in Z_{m}$ and each $k \geqslant m$. The set $C_{\boldsymbol{v}_{0}, k}(a)$ is locally connected continuum as, by 6.3, a union of finitely many topological discs. Each such disc meets any of triangles $T_{v_{k}}$ in an arc (or does not meet at all)

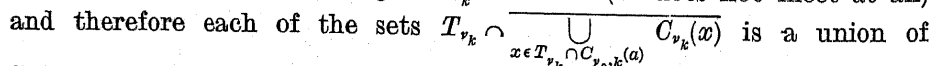
finitely many continuums (actually, each of these sets is a continuum). Since each of these continua is a subset of triangle $T_{v_{k}}$ by definition, then in view of (19) and theorem of Sierpiński (see [4], p. 113) we have (b).
To finish the proof apply theorem of Wilder (see [4], p. 361).

Property (46) is a mere consequence of the two following lemmas: 7.4. For each $l=1,2, \ldots: X-\overline{D_{l}}$ is connected.

For suppose that $X-\overline{D_{l}}$ is not connected between $p$ and $q$ and take, applying 7.3 , simple closed curve $S$ separating the plane between $p$ and $q$. By the definition of $\overline{D_{l}}$ we have

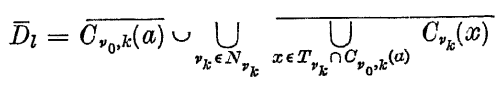

for some $k=0,1, \ldots$ and $a \in Z_{k}$. In view of 6.7 (2), $S$ cannot be contained in $\overline{C_{v_{0}, k}(a)}$ and therefore $S$ must separate one of the sets $X \cap T_{v_{k}}$. Let $C$ be the component of $S \cap \operatorname{Int}\left(T_{\boldsymbol{v}_{k}}\right)$ separating $X \cap \operatorname{Int}\left(T_{\boldsymbol{v}_{k}}\right)$. In view of 6.17

whence

$$
C \subset \operatorname{Int}\left(T_{v_{k}}\right) \cap \bigcup_{x \in T_{v_{k}} \cap C_{v_{0}, k}(a)} \overline{C_{v_{k}}(x)}
$$

$$
\bar{C}=\bigcup_{x \in T_{v_{k}} \cap C_{v_{0} ; k}(a)} \overline{C_{v_{k}}(x)} \cup c_{1} \cup c_{2},
$$

where $c_{1}$ and $c_{2}$ are ends of arc $\bar{C} \subset S$ if $S-C \neq 0$. In view of 6.19 no one of sets $\bar{C} \cap \overline{C_{v_{k}}(x)}, c_{1}$ and $c_{2}$ separates $X \cap T_{v_{k}}$ and therefore at least two of them are non-empty. But it is a contradiction to Sierpinski theorem (see [4], p. 113), because there are only countably many sommands.

7.5. For each $l=1,2, \ldots, X \cap \overline{D_{l}}$ is boundary in $X$.

By the definition of $D_{l}$ and 6.17 we have for some $k=0,1, \ldots$ and

$$
a \in Z_{k}, \overline{D_{l}}=\overline{C_{v_{0}, k}(a)} \cup \bigcup_{v_{k} \in N_{k}} \operatorname{Int}\left(T_{v_{k}}\right) \cap \bigcup_{x \in T_{v_{k}} \cap C_{v_{0}, k}(a)} \overline{C_{v_{k}}(x)},
$$

whence

$$
X \cap D_{l}=X \cap \overline{C_{v_{0}, k}(a)} \cup \bigcup_{v_{k} \in N_{k}} \bigcup_{x \in T_{y_{k}} \cap C_{v_{0}, k}(a)} X \cap \overline{C_{v_{k}}(x)} \text {. }
$$

In view of 6.1 only countably many sets $X \cap \overline{{v_{k}}_{k}(x)}$ differ from the point $x$, and in view of 6.16 all of them are boundary in $X$. And since, in view of 6.7 (1), also $X \cap \overline{{y_{0}, k}(a)}$ is boundary in $X$, then $X \cap \overline{D_{l}}$ as a union of countably many boundary subsets of $X$ is itself boundary in $X$.

Now we proceed to the proper proof of (2), which is based on the two lemmas (compare 7.6 with (3)).

7.6. If continum $S \subset T$ separates $X$ and for some natural $n$ there is $S \cap V_{n}=0$, then $S \cap X$ contains a perfect set.

Proof. Since the set $Z$ contains by its definition the complement of $X$ to $T$, then in view of (44) we can write $S=S \cap X \cup \bigcup_{l=1} S \cap D_{l}$, whence obviously $S=S \cap X \cup \bigcup_{l=1} S \cap \overline{D_{l}}$. 
If $S \cap X$ contains no perfect subset, then ([3], p. 355) it is finite or countable and, a fortiori,

$$
S \cap X-\bigcup_{l=1}^{\infty} S \cap \overline{D_{l}}=\left(p_{1}, p_{2}, \ldots\right) \text {. }
$$

So $S=\bigcup_{j=1}^{\infty}\left\{p_{j}\right\} \cup \bigcup_{l=1}^{\infty} S \cap \overline{D_{l}}$ and from (a), hypothesis $S \cap V_{n}=0$, and (45) we infer that the sommands of $S$ are pairwise disjoint. And because by (46) and (29) no one of them separates $X$ and $S$ does, then at least 2 of them are non-empty. But it is a contradiction to Sierpiński's theorem ([4], p. 133). Hence $S \cap X$ contains a perfect subset.

7.7. If a set $W \subset X-\bigcup_{l=1}^{\infty} V_{l}$ meets each perfect subset $D$ of $X$, then $W \cup V_{n}$ is connected for each $n=1,2, \ldots$

Proof. For supposing that for some natural $n$ the set $W \cup V_{n}$ is not connected, take (11) a continuum $S \subset T$ separating $T$ between 2 points of $W \cup \nabla_{n}$ and such that

(a) $S \cap\left(W \cup V_{n}\right)=0$.

Since $W \cup V_{n} \subset X$, then $S$ would separate also $X$ and therefore. by (a) and 7.6, $S \cap X$ would contain a perfect set $D$. In view of the assumed property of $W$ it implies a contradiction

$$
0 \neq W \cap D \subset W \cap S \cap X \subset W \cap S=0.7
$$

THEOREM 2. The continuum $X$ is a union of a countable sequence of its connected, dense, ponctiform and pairwise disjoint subsets.

Proof. Take a decomposition $X-\bigcup_{l=1}^{\infty} V_{l}=H_{n}$, where sets $H_{n}$ are pairwise disjoint and each of them meets every perfect subset of $X\left({ }^{12}\right)$. In particular, each $H_{n}$ is totally imperfect and

(a) $H_{n}$ is dense in $X$ for each $n=1,2, \ldots$

(i1) We base ourselves here on the following statement: if $A$ is a not connected subset of a topological disc $T$, then there exists continuum $S \subset T$ such that $S \cap A=0$ and $S$ separates $T$ between 2 points of $A$ (cf. [1], p. 233).

(12) The proof of the existence of decomposition $X-\bigcup_{l=1}^{\infty} \nabla_{l}=\bigcup_{n=1}^{\infty} H_{n}$ into pairwise disjoint sets $H_{n}$, each of which meets every perfect subset of $X-\bigcup_{l=1}^{\infty} \nabla_{l}$, strictly follows the Kuratowski's proof of Bernstein theorem (see [3], p. 422). And since, in view of countability of $\bigcup_{l=1}^{\infty} \nabla_{l}$, each perfect subset of $X$ contains a perfect subset of $X-\bigcup_{l=1}^{\infty} \nabla_{l}$, then that decomposition has the property stated above in the proof of theorem.
We show that $\bigcup_{n=1}^{\infty}\left(H_{n} \cup V_{n}\right)$ is the required decomposition of $X$. First of all observe that

$$
\bigcup_{n=1}^{\infty}\left(H_{n} \cup V_{n}\right)=\bigcup_{n=1}^{\infty} H_{n} \cup \bigcup_{n=1}^{\infty} V_{n}=\left(X-\bigcup_{l=1}^{\infty} V_{l}\right) \cup \bigcup_{l=1}^{\infty} V_{l}=X .
$$

Now, by 7.6 , each of the sets $H_{n} \cup \Gamma_{n}$ is connected, and, by (a), each of them is dense in $X$.

As a union of totally imperfect and countable sets, it is ponctiform (see [3], p. 355).

Finally, if $m \neq n$, then $\left(H_{m} \cup \Gamma_{m}\right) \cap\left(H_{n} \cup V_{n}\right)=H_{m} \cap H_{n} \cup H_{m} \cap$ $\cap V_{n} \cup H_{n} \cup V_{m} \cup V_{m} \cap V_{n}$, where first and last sommands are empty, because sets of sequences $\left\{H_{m}\right\}_{m=1,2, \ldots}$ and $\left\{V_{1}\right\}_{l=1,2, \ldots}$ are respectively pairwise disjoint, and also second and third sommands are empty because $H_{m} \cup H_{n} \subset X-\bigcup_{l=1}^{\infty} V_{l}$.

\section{References}

[1] B. Knaster et C. Kuratowski, Sur les ensembles connexes, Fund. Math. 2 (1921), pp. 206-255.

[2] - - A connected and connected in kleinen point set which contains no perfect subset, Bull. of Amer. Math. Soe. 33 (1927), p. 106.

[3] C. Kuratowski, Topologie I, Warszawa 1948.

[4] - Topologie II, Warszawa 1952.

[5] A. Lelek, Ensembles $\sigma$-connexes et le théorème de Gehman, Fund. Math. 47 (1959), pp. 265-276.

[6] G. T. Whyburn, Analytic topology, New York 1942.

INSTITUTE OF MATHEMATICS OF THE POLISH ACADEMY OF SCIENCES GONVILLE AND CAIUS COLLEGE, CAMBRIDGE

Reçu par la Rédaction le 21.5.1964 